\title{
On Heatshield Shapes for Mars Entry Capsules
}

\author{
Dinesh K. Prabhu ${ }^{1}$ and David A. Saunders ${ }^{2}$ \\ ERC, Inc., NASA Ames Research Center, Moffett Field, CA, 94035
}

The $70^{\circ}$ sphere-cone - the standard geometry for all US Mars entry missions - is thoroughly examined via flow field simulations at a select few peak heating points along candidate flight trajectories. Emphasis is placed on turbulent heating based on the BaldwinLomax turbulence model. It is shown that increased leeward turbulent heating for a $\mathbf{7 0}^{\circ}$ sphere-cone flying at angle of attack is primarily due to the discontinuity in curvature between the spherical nose cap and the conical frustum - the attachment of the sonic line at this sphere-cone junction leads to a supersonic edge Mach number over the leeward acreage. In an attempt to mitigate this problem of elevated turbulent heating, alternate geometries, without any curvature discontinuities in the acreage, are developed. Two approaches, one based on nonlinear optimization with constraints, and one based on the use of non-uniform rational B-splines, are considered. All configurations examined remain axisymmetric. The aerothermal performance of alternate geometries is shown to be superior to that of the $70^{\circ}$ sphere-cone.

\section{Nomenclature}

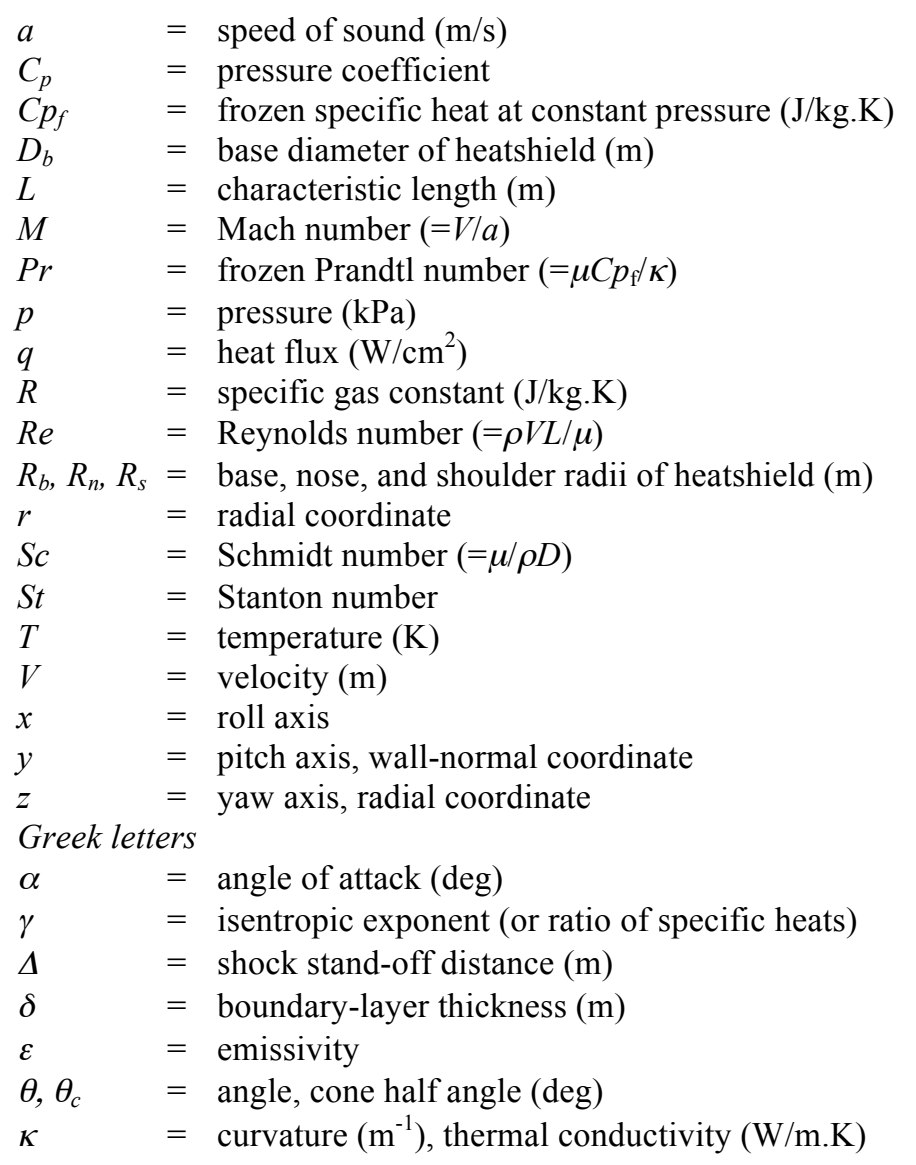

\footnotetext{
${ }^{1}$ Senior Research Scientist, Aerothermodynamics Branch, 230-3, and Associate Fellow AIAA.

${ }^{2}$ Senior Research Scientist, Aerothermodynamics Branch, 230-2, and Senior Member AIAA.
} 


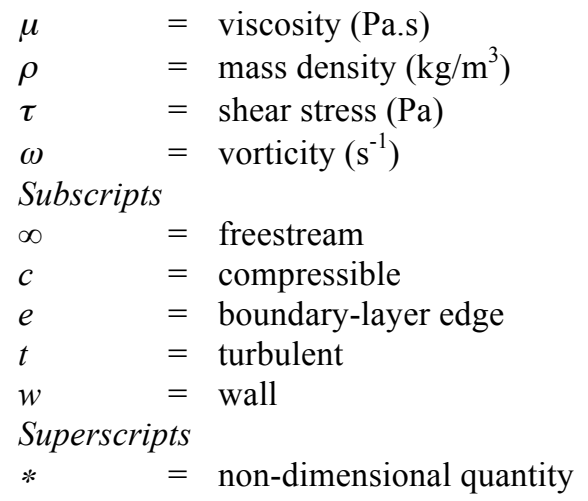

\section{Introduction}

T HE success of NASA's Viking mission in the 1970s has meant that all subsequent missions to Mars have relied on a $70^{\circ}$ sphere-cone capsule configuration to land scientific experiments on the surface of the planet; staying with a proven architecture/configuration carries less risk. The paper of Braun and Manning ${ }^{1}$ provides a good summary of all NASA missions to Mars starting with Viking, and the challenges of entry, descent and landing (EDL) at Mars. Table 1 lists the entry type and heatshield geometries (along with key parameters) of capsules used by the United States (US) in several Mars missions from 1975 through 2011. The data have been extracted from the compilation of Davies and Arcadi. ${ }^{2}$

Table 1. Heatshield parameters for several US Mars missions.

\begin{tabular}{lccccccc}
\hline \hline & Entry Type & HS Geometry & $\begin{array}{c}\boldsymbol{D}_{\boldsymbol{b}} \\
\mathrm{m}\end{array}$ & $\begin{array}{c}\boldsymbol{R}_{\boldsymbol{n}} \\
\mathrm{m}\end{array}$ & $\begin{array}{c}\boldsymbol{R}_{\boldsymbol{s}} \\
\mathrm{m}\end{array}$ & $\boldsymbol{R}_{\boldsymbol{n}} / \boldsymbol{R}_{\boldsymbol{b}}$ & $\boldsymbol{R}_{\boldsymbol{s}} / \boldsymbol{R}_{\boldsymbol{b}}$ \\
\hline${\text { Deep Space } 2^{\mathrm{a}}}$ & & & & \\
Viking & Ballistic & $45^{\circ}$ Sphere-cone & 0.35 & 0.088 & & 0.500 & \\
Mars Pathfinder & Lifting & $70^{\circ}$ Sphere-cone & 3.54 & 0.893 & 0.025 & 0.500 & 0.014 \\
Mars Exploration Rovers & Ballistic & $70^{\circ}$ Sphere-cone & 2.65 & 0.663 & & 0.500 & \\
Mars Phoenix & Ballistic & $70^{\circ}$ Sphere-cone & 2.65 & 0.663 & & 0.500 & \\
Mars Science Laboratory $^{\mathrm{b}}$ & Ballistic & $70^{\circ}$ Sphere-cone & 2.65 & 0.663 & 0.066 & 0.500 & 0.050 \\
\hline \hline
\end{tabular}

${ }^{\mathrm{a}}$ Mission presumed failed

${ }^{b}$ During writing of the present paper, the MSL vehicle was launched successfully on Nov. 26, 2011

The latest in the series of Mars missions is the Mars Science Laboratory (MSL), which was launched on November 26, 2011. ${ }^{3}$ This $4.5 \mathrm{~m}$ diameter $70^{\circ}$ sphere-cone configuration will be NASA's largest capsule to enter the Mars atmosphere (or any planetary atmosphere for that matter). Like Viking which flew a lifting trajectory with a nominal angle of attack of $-11^{\circ}$, the MSL capsule too will fly a lifting trajectory, only this time with a nominal angle of attack of $-16^{\circ}$.

As with any blunt capsule at angle of attack, larger size means longer running lengths of streamlines (from the stagnation point) towards the leeward shoulder and hence increased likelihood of transition to turbulence, with a consequent increase in heating on the leeward side. ${ }^{4}$ For certain regions of the flight trajectory space (parameterized by entry velocity and entry flight path angle), MSL is likely to experience significant turbulent heating, a situation that will be further exacerbated by surface roughness that develops due to ablation of the Thermal Protection System (TPS). To illustrate the nature of the problem, contours of computed (and unmargined) surface turbulent heat flux, along with centerline distributions at the peak heating point along a flight trajectory for a $4.7 \mathrm{~m}$ diameter $70^{\circ}$ spherecone (a candidate geometry for a future Mars mission) are shown in Fig. 1a and Fig. 1b. On the leeward side, the elevated levels of turbulent heating, as much as 6 to 7 times the corresponding laminar value, are clearly seen. In the case of MSL, this likelihood of transition and heating augmentation due to surface roughness, coupled with material failure observed during arc-jet testing, ${ }^{5}$ led the project to abandon SLA-561V (Super Light Ablator) as the first choice of thermal protection material with Mars Pathfinder flight heritage, and use PICA (Phenolic-Impregnated Ceramic Ablator) instead. ${ }^{6}$

Despite mitigating the risk associated with turbulent heating, one question that has been left unanswered in the MSL project is: are there other heatshield shapes that result in lower turbulent heating on the leeside? There is currently a debate within the US Mars program community on a change to the heatshield shape. The first choice for 
a new heatshield for Mars entry is naturally one with flight heritage (albeit for Earth entry), and that is a spherical section heatshield used in several space programs including Apollo and Orion.

In an attempt to answer the question yet unanswered by the Mars program, a fresh look at heatshield shapes has been taken in the present work, and a few "non-traditional" (yet analytic) shapes have been developed and analyzed using modern flow field simulation tools. ${ }^{7,8}$ It should be noted that the idea of moving away from "traditional" capsule shapes - sphere-cones or spherical sections - is not new. For instance, as part of NASA's Exploration Systems Architecture Study (ESAS), ${ }^{9}$ asymmetric capsule shapes were developed and studied. ${ }^{10}$ Although the "traditional" symmetric spherical section heatshield (Apollo shape) was finally selected for the Orion capsule, the groundwork had been laid for systematic development and analysis of asymmetric configurations defined by a finite number of geometric parameters. The present paper builds upon this foundation, and new symmetric shapes that lie between a sphere-cone and a completely spherical section are developed and studied. Retaining axisymmetry still leaves plenty of room for variation, so any possible benefits of asymmetry are not pursued here. The focus of the present computational study is only on the aerothermodynamic environments for these new shapes. It is hoped that these configurations, after more experimental testing, will be considered as possible choices for future MSL-class Mars missions, or perhaps as starting points for more elaborate shape optimization studies.

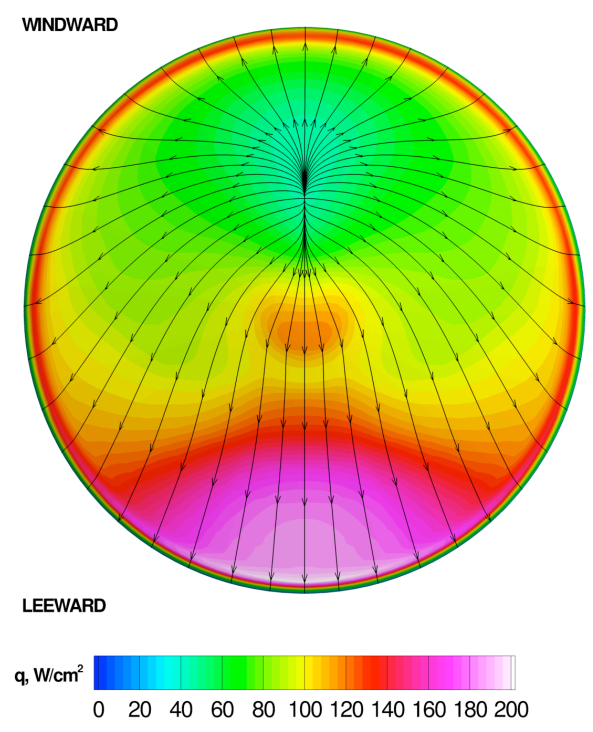

(a) Surface heat flux

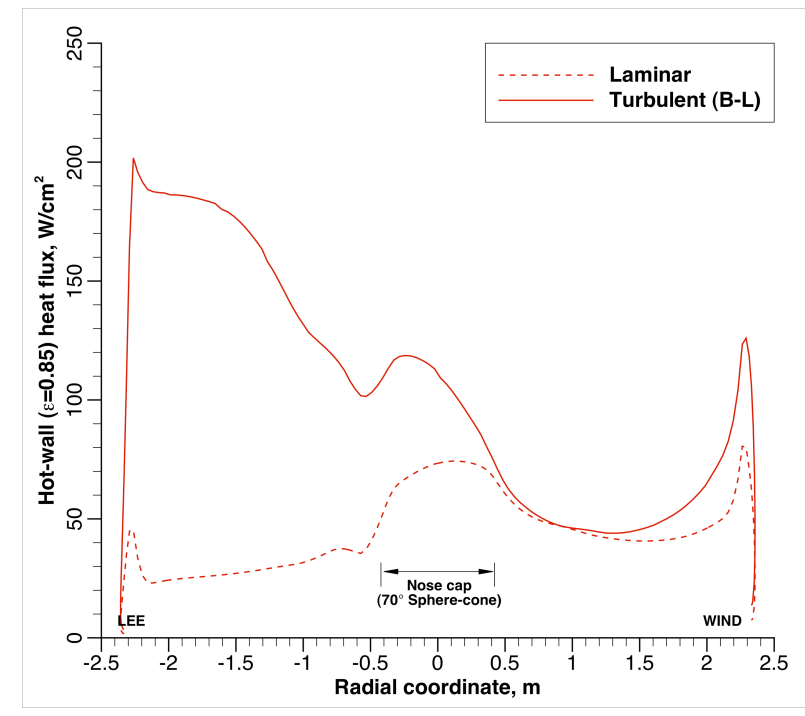

(b) Centerline heat flux

Figure 1. Surface and centerline distributions of hot-wall $(\varepsilon=0.85)$ heat flux for a $70^{\circ}$ sphere-cone at $-15.7^{\circ}$ angle of attack for a freestream velocity of $5.41 \mathrm{~km} / \mathrm{s}$. Elevated levels of turbulent heating (Baldwin-Lomax model) on the leeward side are clearly seen. Results are for a smooth wall, and the wall is assumed "supercatalytic" to recombination.

\section{Ground Rules and Assumptions}

\section{A. Geometries}

As already indicated, only axisymmetric forebody configurations are considered in the present work. This choice simplifies the construction of three-dimensional candidate heatshield geometries. One only needs to construct the generatrix (a 2D plane curve) and simply rotate that generatrix around the $x$-axis (considered the capsule roll axis in the present work) to construct the three-dimensional surface representing the outer mold line (OML) of the heatshield. The two most commonly used geometries for axisymmetric heatshields of atmospheric entry capsules are the sphere-cone and the spherical section whose generatrices are respectively shown in Figs. 2a and 2b.

The generatrix of a sphere-cone heatshield (Fig. 2a) is actually composed of three segments - a circle segment for the nose, a straight line segment for the cone, and another circle segment for the shoulder - which are blended smoothly, i.e., there is no discontinuity in slope where any two segments meet. This composite plane curve is completely described by four parameters $-R_{b}, R_{n}, R_{s}$, and $\theta_{c}$ - where $R_{n}<R_{b}, R_{s}<<R_{b}$ (or $R_{n}$ ), and $0^{\circ}<\theta_{c}<90^{\circ}$ ( $70^{\circ}$ is usually the limit!). 
The generatrix of a spherical section heatshield is composed of two segments (Fig. 2b) - a circle segment representing the dish, and a circle segment representing the shoulder. Again, the two segments are blended smoothly at the dish-shoulder junction. This second composite plane curve is completely described by three parameters $-R_{b}$, $R_{n}$, and $R_{s}-$ where $R_{n}>R_{b}$, and $R_{s}<<R_{b}$ (or $\left.R_{n}\right)$.

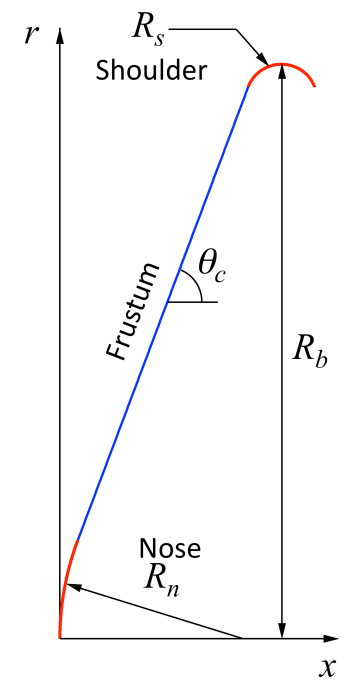

(a) Sphere-cone

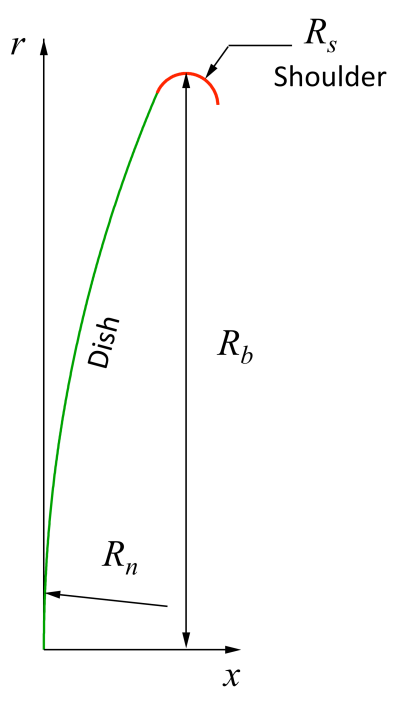

(b) Spherical section

Figure 2. Generatrices of typical symmetric heatshield geometries: (a) a sphere-cone parameterized by the radius, $R_{n}\left(R_{n}<R_{b}\right)$, of the nosecap, the radius, $R_{s}\left(R_{s}<<R_{b}\right.$ or $\left.R_{n}\right)$, of the shoulder torus, and the cone half angle, $\theta_{c}\left(0^{\circ}<\theta_{c}<90^{\circ}\right)$; and (b) a spherical section parameterized by the radius, $R_{n}\left(R_{n}>R_{b}\right)$, of the dish, and the radius, $R_{s}\left(R_{s}<<R_{b}\right.$ or $\left.R_{n}\right)$, of the shoulder torus. The base radius for either geometry is $R_{b}$.

Both the composite curves shown in Figs. $2 \mathrm{a}$ and $2 \mathrm{~b}$ are simple enough that analytical expressions for the coordinates of points of intersection of the component segments can be obtained with straightforward algebra. For instance, if the apex of the sphere-cone heatshield generatrix (Fig. 2a) is at the origin $(0,0)$ of the coordinate system $(x, r)$, then the coordinates $\left(x_{t, n}, r_{t, n}\right)$ of the point of tangency between the circle and the straight line (inclined at $\theta_{c}$ to the $x$-axis) are:

$$
x_{t, n}=R_{n}\left[1-\sin \theta_{c}\right] \quad r_{t, n}=R_{n} \cos \theta_{c}
$$

and the coordinates $\left(x_{t, s}, r_{t, s}\right)$ of the point of tangency of the straight line and the shoulder circle are:

$$
x_{t, s}=R_{n}\left(1-\sin \theta_{c}\right)+\left[R_{b}-R_{n} \cos \theta_{c}-R_{s}\left(1-\cos \theta_{c}\right)\right] \cot \theta_{c} \quad r_{t, s}=R_{b}-R_{s}\left[1-\cos \theta_{c}\right]
$$

Similarly, for the spherical section generatrix (Fig. 2b), the coordinates $\left(x_{t, s}, r_{t, s}\right)$ of the tangency point between the circle segment representing the dish and the circle segment representing the shoulder are:

$$
x_{t, s}=R_{n}\left(1-\frac{\sqrt{\left(R_{n}-R_{b}\right)\left(R_{n}+R_{b}-2 R_{s}\right)}}{R_{n}-R_{s}}\right) \quad r_{t, s}=R_{n} \frac{R_{b}-R_{s}}{R_{n}-R_{s}}
$$

The angle, $\theta$, between the $x$-axis and the tangent to the dish at the intersection point with the shoulder is:

$$
\theta=\arccos \left(\frac{R_{b}-R_{s}}{R_{n}-R_{s}}\right)
$$

An important geometric aspect of a heatshield, and one often overlooked in flowfield analysis, is the distribution of curvature of the three-dimensional surface. The choice of axisymmetric shapes considerably simplifies the 
determination of curvature. One needs to consider only the radial distribution of the one-dimensional curvature of the generatrix (a plane curve), i.e., the longitudinal curvature of the three-dimensional surface swept by the generatrix; the other principal curvature of the surface, the one in the circumferential direction, is a constant for a given $x$ value due to the choice of axially symmetric shapes. The radial distribution of one-dimensional curvature (magnitude) of the generatrix is:

$$
\kappa(r)=\left|\frac{d^{2} x}{d r^{2}}\left[1+\left(\frac{d x}{d r}\right)^{2}\right]^{-\frac{3}{2}}\right|
$$

The distributions of curvature for the two most common generatrices, the sphere-cone and the spherical section (Figs. 2a and 2b), are shown in Fig. 3.

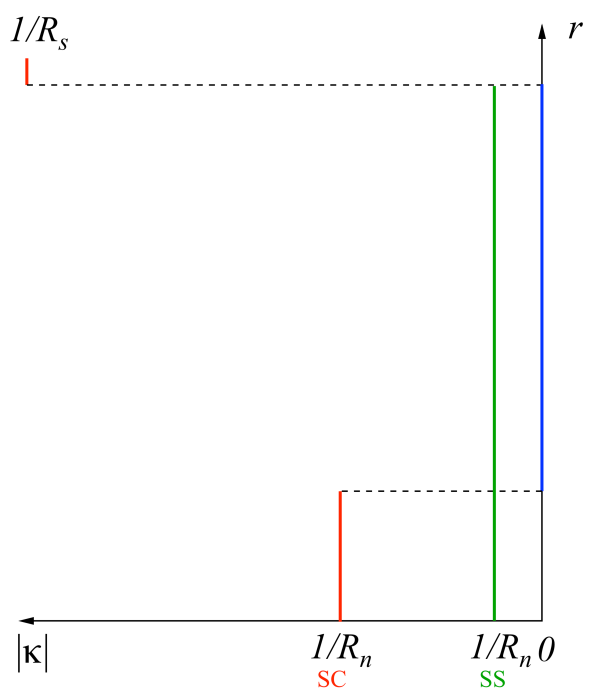

Figure 3. Curvature $(\kappa)$ distributions of sphere-cone and spherical section geometries. The abbreviation SC stands for sphere-cone, and SS stands for spherical section. For either geometry, curvature changes discontinuously at the points of tangency between component segments.

It should be noted that while the geometric construction (Eq. 1 or Eq. 2) guarantees continuity of the first derivative in $r$ between various segments of the heatshield, it does not guarantee continuity in the second derivative, and hence, curvature. It is hypothesized that a discontinuity in the second derivative would have an impact on predictions of surface aerothermal environments for these traditional shapes. This hypothesis is put to the test in the present paper.

From Table 1 it is evident that, with the sole exception of Deep Space 2, all US Mars missions with an atmospheric entry component (lifting or ballistic) in the mission profile have used a $70^{\circ}$ sphere-cone geometry for the heatshield. The $70^{\circ}$ sphere-cone shape has the high drag necessary to decelerate the capsule through the tenuous Martian atmosphere. Therefore, the point of departure for a heatshield shape study - the focus of the present paper is a $70^{\circ}$ sphere-cone.

For all the cases listed in Table 1, the radius of the spherical nose cap is always one half the base radius, i.e., $R_{n} / R_{b}=0.5$, and the shoulder radius is roughly one tenth that of the nose, i.e., $R_{s} / R_{n} \approx 0.1$ or $R_{s} / R_{b} \approx 0.05$. There is no information in the extant literature for the rationale behind the particular values of these ratios, especially $R_{n} / R_{b}$. The influence of these ratios is also explored in the present work.

Although a spherical section heatshield has never been used in US Mars missions, such a shape is the baseline for human-rated Earth-entry capsules such as Mercury, Gemini, Apollo, and the current Multi-Purpose Crew Vehicle (MPCV). Therefore, the spherical section heatshield is considered as an alternate baseline in the present work. The aerothermal performance of other symmetric shapes developed in the following sections is gauged against these two shapes with flight heritage. 


\section{B. Modeling and simulation}

The modeling assumptions used in the present study are identical to those used in the development of aerothermal design environments for MSL. ${ }^{3}$ Key modeling assumptions used in $\operatorname{LAURA}^{6}$ and $\operatorname{DPLR}^{7}$ simulations for MSL are summarized in Table 2. In the present work version 4.02.2 DPLR is used in all simulations; v3.05 of DPLR was used in MSL simulations.

Table 2. Models used in flow field simulations with v4.02.2 of DPLR.

\begin{tabular}{|c|c|}
\hline Model & Details \\
\hline Gas mixture & $\begin{array}{l}\text { - 8-species }\left(\mathrm{CO}_{2}, \mathrm{CO}, \mathrm{N}_{2}, \mathrm{O}_{2}, \mathrm{NO}, \mathrm{C}, \mathrm{N} \text {, and } \mathrm{O}\right) \text { Mars gas mixture } \\
\text { - A } 97-3 \% \text { mixture, by mass, of } \mathrm{CO}_{2}-\mathrm{N}_{2} \text { is assumed as the freestream }\end{array}$ \\
\hline Thermodynamics & $\begin{array}{l}\text { - Swo-temperature }\left(T-T_{v}\right) \text { model } \\
\text { - } \text { each molecular species } \\
\text { - Excited electronic states are also included for all species (atoms and molecules) }\end{array}$ \\
\hline Laminar transport & $\begin{array}{l}\text { - Collision-integrals for species pairs with Gupta-Yos mixing rule } \\
\text { - Mass diffusion modeled by SCEBD (Self-Consistent Effective Binary Diffusion) } \\
\text { - The SCEBD model, an approximation to a true multi-component diffusion model, } \\
\text { does not require specification of a Schmidt number }\end{array}$ \\
\hline Turbulent transport & $\begin{array}{l}\text { - Baldwin-Lomax algebraic model including modifications for compressibility and } \\
\text { streamwise pressure gradient } \\
\text { - Prandtl number }\left(P r_{t}\right)=0.9 \\
\text { - Schmidt number }\left(S c_{t}\right)=0.7\end{array}$ \\
\hline Reaction mechanism \& rates & $\begin{array}{l}\text { - Mitcheltree mechanism } \\
\text { - Rates from Park et al. }\end{array}$ \\
\hline Wall boundary condition & $\begin{array}{l}\text { - "Supercatalytic" to recombination } \\
\text { - "Supercatalytic" means that the freestream composition is recovered at the wall, i.e., } \\
\text { - Ririchlet boundary conditions are used for mass fractions at the wall } \\
\text { - Energy balance between heat brought to the wall by convection and diffusion and re- } \\
\text { radiated into free space (at } 0 \mathrm{~K} \text { ). No heat conduction through the thickness of the } \\
\text { material }\end{array}$ \\
\hline
\end{tabular}

The turbulence modeling aspect requires a little further elaboration. The turbulence model used in DPLR simulations for MSL and other geometries discussed here is the zero-equation algebraic model of Baldwin and Lomax. ${ }^{11}$ This model, originally developed for incompressible flows, has been modified to account for flow compressibility ${ }^{12}$ and the influence of adverse pressure gradients in the stream direction. ${ }^{13}$ These two modifications to the inner layer of the Baldwin-Lomax model are:

$$
\eta_{c}^{+}=\eta \frac{\sqrt{\rho \tau_{w}}}{\mu} \quad A_{p}^{+}=A^{+} \sqrt{\frac{\tau_{w}}{\tau}}=A^{+} \sqrt{\frac{\tau_{w}}{\left(\mu+\mu_{t}\right)|\omega|}} \quad A^{+}=26
$$

where $\eta$ is the directed distance from the wall, $\tau_{w}$ is the shear stress at the wall, $\mu_{t}$ is the turbulent eddy viscosity, and $|\omega|$ is the magnitude of vorticity. It should be noted that the turbulent eddy viscosity enters into the model implicitly in the modified definition of $A^{+}$.

The influence of compressibility is accounted for when local values (instead of wall values) of $\rho$ and $\mu$ are used in the definition of $\eta^{+}$. The influence of the streamwise pressure gradient comes in through the parameter $A^{+}$, which usually has a value of 26 . It is scaled by the ratio of the shear stress, $\tau_{w}$, at the wall to the local value, $\tau$. The modified $\eta^{+}$and $A^{+}$are then used in the Van Driest damping function, $D$ :

$$
D=\left[1-\exp \left(-\frac{\eta_{c}^{+}}{A_{p}^{+}}\right)\right] \quad l_{m i x}=\kappa \eta D \quad \kappa=0.4
$$

Although the correction for compressibility has an influence on the magnitude of $D$ in Eq. 5, the influence of the pressure gradient correction term is larger, especially since it includes the magnitude of local vorticity and the local turbulent eddy viscosity. Any decrease in the exponential term means increased mixing length, and hence increased turbulent eddy viscosity because the turbulent eddy viscosity depends on the square of the mixing length. 
Furthermore, depending on the curvature of the shock, its standoff from the body, and the Reynolds number (which dictates the thickness of the wall-bounded shear layer), the attached shear layer can entrain rotational flow generated by a curved shock (Crocco's theorem ${ }^{14}$ ), and hence cause enhanced heating. A second line of inquiry in the present paper is the interplay between the turbulence model and discontinuities in surface curvature.

\section{Flow conditions for flight simulations}

It is well known that the maximum predicted heat flux, with appropriate margins to account for uncertainties in modeling and simulation, along a flight trajectory dictates the choice of TPS material for the heatshield. ${ }^{15}$ Therefore, only peak heating points along a few design trajectories for a proposed 2018 US mission to Mars are considered in the present work. The freestream conditions necessary for CFD simulations at these points are given in Table 3 . The thickness of the chosen TPS material, which is dependent on the time-integrated value of heat flux (i.e., heat load) over a trajectory, is not considered here.

Table 3. Freestream conditions chosen for present study.

\begin{tabular}{lcccccccc}
\hline \hline Trajectory & $\begin{array}{c}\boldsymbol{V}_{\infty} \\
\mathrm{m} / \mathrm{s}\end{array}$ & $\begin{array}{c}\boldsymbol{\alpha} \\
\mathrm{deg}\end{array}$ & $\begin{array}{c}\boldsymbol{\rho}_{\infty} \\
\mathrm{kg} / \mathrm{m}^{3} \\
\times 10^{4}\end{array}$ & $\begin{array}{c}\boldsymbol{T}_{\infty} \\
\mathrm{K}\end{array}$ & $\boldsymbol{M}_{\infty}$ & $\begin{array}{c}\boldsymbol{R} \boldsymbol{e}_{\infty} \\
1 / \mathrm{m} \\
\times 10^{6}\end{array}$ & $\begin{array}{c}\boldsymbol{Q}_{\infty} \\
\mathrm{kPa}\end{array}$ & $\begin{array}{c}\boldsymbol{q}_{\infty}{ }^{\mathrm{a}} \\
\mathrm{W} / \mathrm{cm}^{2}\end{array}$ \\
\hline Retrograde/Steep & 5411.2 & -15.71 & 10.13 & 182.6 & 24.2 & 0.585 & 14.83 & 96 \\
Prograde/Nominal & 5070.9 & -15.54 & 9.06 & 175.9 & 23.3 & 0.510 & 11.65 & 75 \\
Prograde/Shallow & 5165.0 & -15.54 & 6.87 & 168.8 & 24.2 & 0.411 & 9.16 & 69 \\
\hline \hline
\end{tabular}

${ }^{a}$ Cold-wall heat flux, computed using Sutton-Graves formula [Ref. 16], at the stagnation point of a hemisphere of $1 \mathrm{~m}$ radius

\section{Preliminary computations with traditional shapes}

First, baseline heating environments are established for the two candidate geometries - a $70^{\circ}$ sphere-cone geometry and a spherical section geometry. Both heatshield geometries have a base diameter of $4.7 \mathrm{~m}$, a nearly $5 \%$ increase over the diameter of MSL. At the time when the present study was undertaken, mission planners had considered an increase over the diameter of the MSL geometry to accommodate a larger payload.

Next, since the focus of the present paper is on turbulent heating over the heatshield, we calibrate the simulation tool - v4.02.2 of DPLR - to ground-based turbulent flow experiments (with and without any real-gas effects). Having calibrated the simulation tool, we then systematically examine the influence of real-gas effects, bluntness, curvature continuity (or lack thereof), angle of attack, freestream conditions, etc., on turbulent heating before developing alternate geometries for improved aerothermodynamic performance of the heatshield under turbulent flow conditions.

\section{A. Establishing the baseline: $70^{\circ}$ sphere-cone vs spherical section}

For the peak heating point along a retrograde/steep entry trajectory (Table 5), laminar and turbulent heating computations are performed for both the $70^{\circ}$ sphere-cone and spherical section geometries. The centerline distributions of hot-wall $(\varepsilon=0.85)$ heat flux and pressure for the two geometries are shown in Fig. 4. 


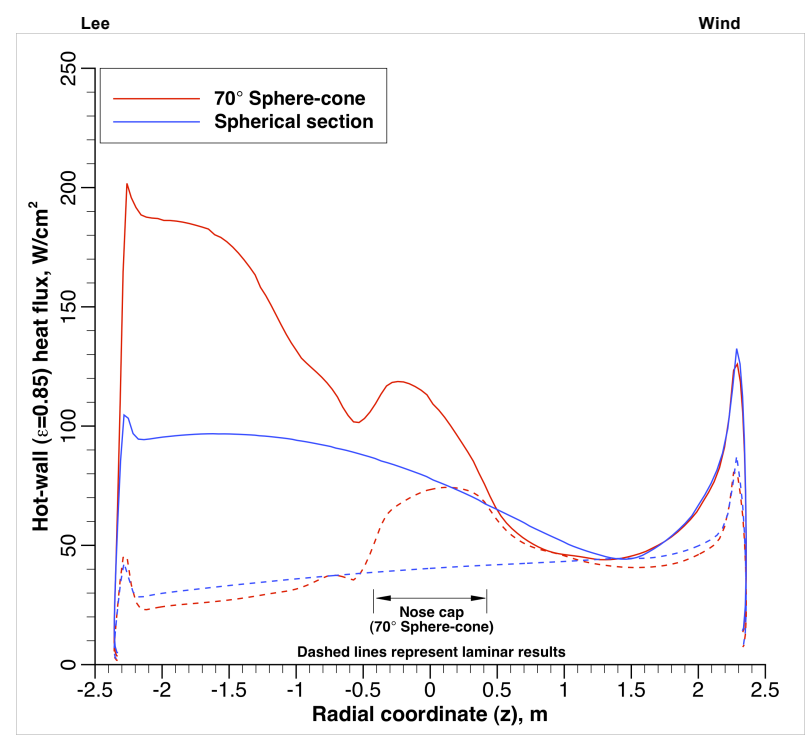

(a) Heat flux

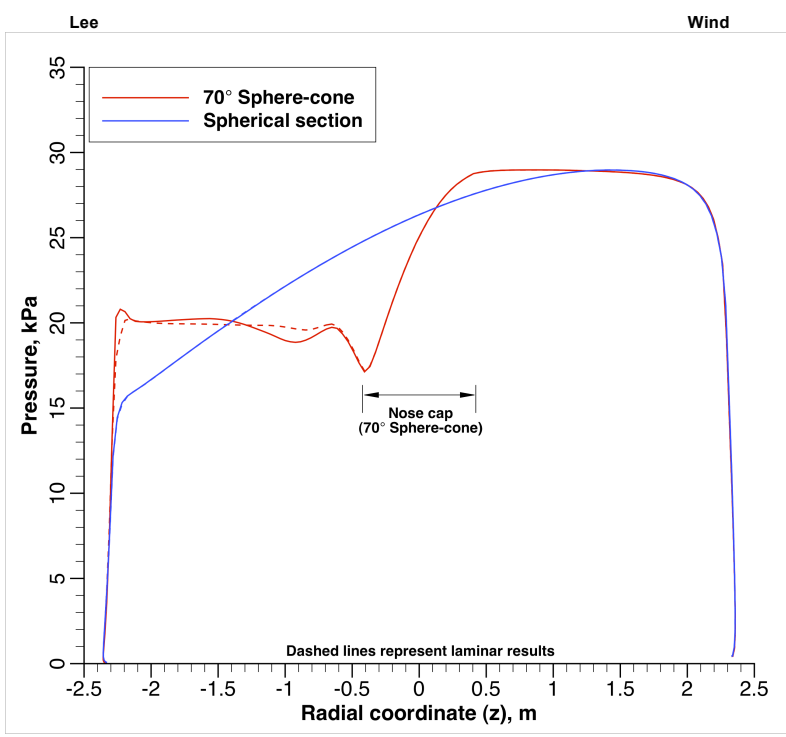

(b) Pressure

Figure 4. Centerline distributions of hot-wall $(\varepsilon=0.85)$ heat flux and pressure for a $70^{\circ}$ sphere-cone and a spherical section heatshield (both $4.7 \mathrm{~m}$ in diameter). Laminar and turbulent (Baldwin-Lomax) results are shown for a flight speed of $5.41 \mathrm{~km} / \mathrm{s}$ and an angle of attack of $-15.7^{\circ}$. Dashed lines represent laminar results.

For the laminar case, the spherical section has heating levels that are roughly no more than $15 \%$ of the levels on the windward and leeward flanks of the cone (Fig. 4a). However, the spherical nose of the cone experiences much larger heating. For the turbulent case, the spherical section experiences much less heating (about $50 \%$ less) over much of the leeward acreage. Unlike the spherical section, which has a smooth distribution of turbulent heating, the sphere-cone experiences a very rapid rise in turbulent heating level past the sphere-cone junction on the leeward side.

A probable cause for this lies in the pressure distributions shown in Fig. 4b. The spherical section has a favorable pressure gradient over the entire wetted acreage, while the sphere-cone pressure distribution is influenced by the curvature discontinuity at the junction of the spherical nose cap and the conical frustum. Furthermore, the laminar and turbulent pressure distributions towards the leeward side differ significantly in character for the sphere-cone geometry compared to the spherical section, which does not show a difference in pressure distributions for laminar and turbulent flow. For the $70^{\circ}$ sphere-cone geometry, the laminar pressure distribution beyond the point of curvature inflection is smoother on the leeward side, but the pressure gradient appears to be somewhat unfavorable in the streamwise direction. The turbulent pressure distribution, on the other hand, has multiple inflection points towards the leeward shoulder. These inflection points appear to line up with rate of change of heat flux towards the leeward shoulder. This behavior of surface pressure is of great interest, and is investigated systematically in the present section.

It is first necessary to examine available experimental data obtained from various ground-based facilities to see if the measured and predicted heating distributions correlate with the predicted pressure distributions and inflections (if any) in them. For the $70^{\circ}$ sphere-cone geometry, there is a wealth of aerothermal testing data acquired under the MSL program. There is also a wealth of test data acquired for the spherical section under the MPCV (Multi-Purpose Crew Vehicle) program. However, the spherical section geometry developed under the Mars 2018 program differs from that of the MPCV in terms of the dish radius, $R_{n}$, and the shoulder radius, $R_{s}$. Therefore, there are no experimental data for that particular shape.

\section{B. Ground-based experiments}

The first test case, that of high Reynolds number flow of pure $\mathrm{N}_{2}$, is one taken from a matrix of conditions presented in the comprehensive review paper of Hollis. ${ }^{17}$ The freestream conditions for this case are presented in Table 4. At these conditions, a $15.24 \mathrm{~cm}$ diameter $70^{\circ}$ sphere-cone $(0.0339$-scale MSL) and a $17.78 \mathrm{~cm}$ diameter spherical section (0.0354-scale MPCV) were tested in the T9 tunnel (a conventional blowdown tunnel) at Arnold Engineering Development Center (AEDC) in Silver Spring, MD. The angles of attack for the two shapes, however, 
were not identical in the tests - the $70^{\circ}$ sphere-cone was tested at an angle of attack of $-16^{\circ}$, while the spherical section was tested at an angle of attack of $-28^{\circ}$. Although the freestream Reynolds number is high enough to guarantee fully turbulent flow over the entire heatshield (see Ref. 17) for both shapes, the stagnation enthalpy of the flow is quite low. Therefore, the simulations of this case address only the fluid mechanical aspects of the turbulent flow problem and are not encumbered by any real-gas effects.

Table 4. Freestream condition for a case from the AEDC T9 Tunnel MSL/MPCV test matrix.

\begin{tabular}{cccccccc}
\hline \hline $\begin{array}{c}\text { Test } \\
\text { Gas }\end{array}$ & $\boldsymbol{M}_{\infty}$ & $\begin{array}{c}\boldsymbol{R e}_{\infty} \times \mathbf{1 0}^{-7} \\
(1 / \mathrm{m})\end{array}$ & $\begin{array}{c}\boldsymbol{p}_{\infty} \\
(\mathrm{kPa})\end{array}$ & $\begin{array}{c}\boldsymbol{T}_{\infty} \\
(\mathrm{K})\end{array}$ & $\begin{array}{c}\boldsymbol{\rho}_{\infty} \\
\left(\mathrm{kg} / \mathrm{m}^{3}\right)\end{array}$ & $\begin{array}{c}\boldsymbol{U}_{\infty} \\
(\mathrm{m} / \mathrm{s})\end{array}$ & $\begin{array}{c}\boldsymbol{H}_{\mathbf{0}} \boldsymbol{h}_{\boldsymbol{w}}, \mathbf{3 0 0 K} \\
(\mathrm{MJ} / \mathrm{kg})\end{array}$ \\
\hline $\mathrm{N}_{2}$ & 8.0 & 16 & 11.8553 & 70.1 & 0.5718 & 1356.8 & 0.681 \\
\hline \hline
\end{tabular}

For the freestream condition given in Table 4, flow computations are performed, first assuming the flow to be completely laminar, and then assuming the flow to be fully turbulent. In all computations, the wall is assumed isothermal with a temperature of $300 \mathrm{~K}$. Since the flow is of pure $\mathrm{N}_{2}$ and the stagnation enthalpy, $H_{0}$, is low, computations are performed with $\gamma=1.4$ and a specific gas constant, $R$, of $296.78 \mathrm{~J} / \mathrm{kg} . \mathrm{K}$. Sutherland's law is used for molecular viscosity, and the thermal conductivity of the gas is computed with the assumption of a constant Prandtl number $(P r=0.71)$. The Baldwin-Lomax algebraic turbulence model, corrected for compressibility effects and streamwise pressure gradient, is used in the turbulent flow simulations. It is assumed that the flow transitions from laminar to turbulent at the stagnation point, and therefore, no model for transition is employed in the turbulent flow simulations.

The predicted centerline distributions of cold-wall heat flux (laminar and turbulent) are compared against experimental data in Figs. $5 \mathrm{a}$ and $5 \mathrm{~b}$ for the two shapes, $70^{\circ}$ sphere-cone and spherical section, respectively. Both predictions and experimental data are nondimensionalized as:

$$
z^{*}=\frac{z}{R_{b}}, \quad q^{*}=S t \sqrt{R e_{\infty, D}}=\frac{(\gamma-1) q}{\gamma \rho_{\infty} R\left(H_{0}-h_{w, 300 \mathrm{~K}}\right)} \sqrt{\frac{\rho_{\infty} U_{\infty} D}{\mu_{\infty}}}, \quad p^{*}=\frac{p}{\frac{1}{2} \rho_{\infty} U_{\infty}^{2}}
$$

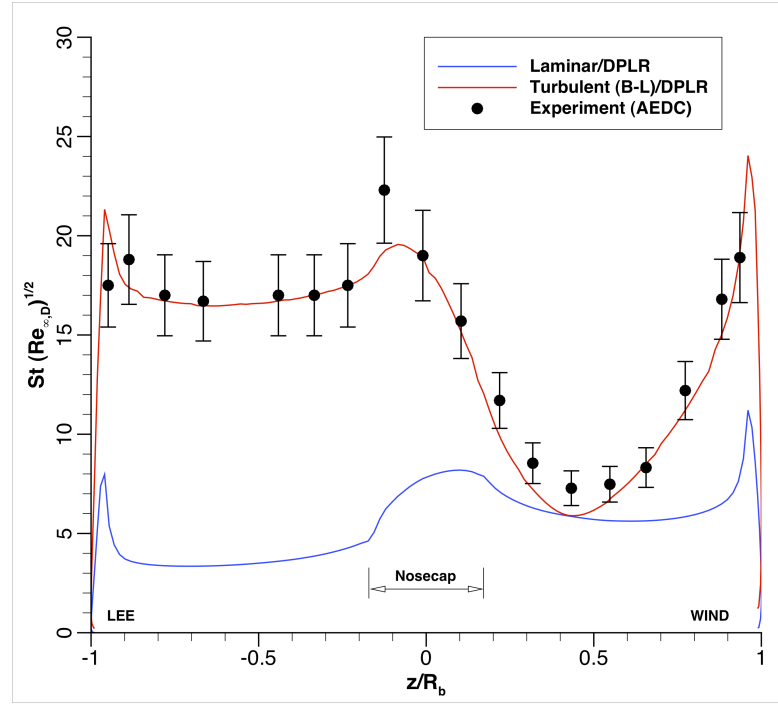

(a) $70^{\circ}$ sphere-cone, $\alpha=-16^{\circ}$

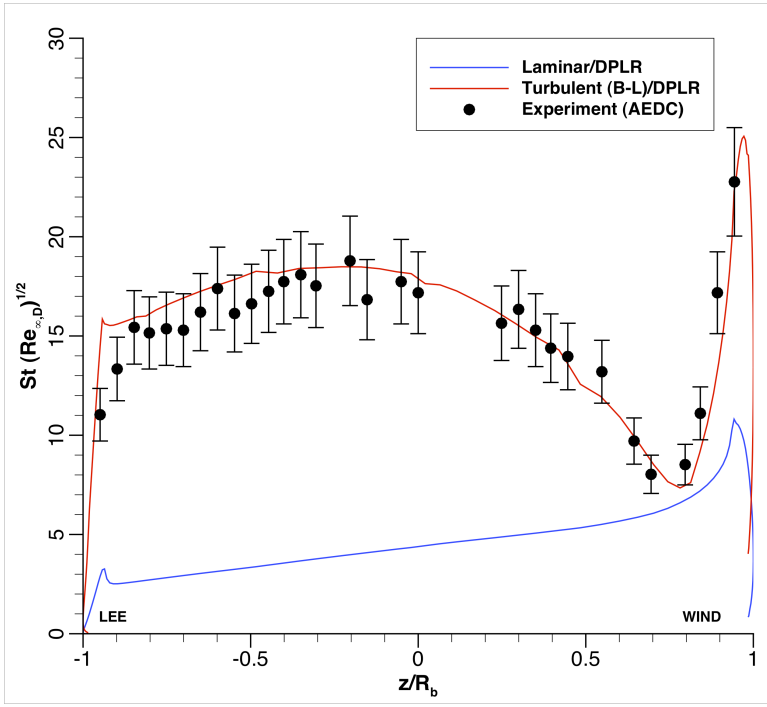

(b) Spherical section, $\alpha=-28^{\circ}$

Figure 5. Centerline non-dimensional cold-wall $\left(T_{w}=300 \mathrm{~K}\right)$ heat flux (or equivalently, scaled Stanton number) distributions for: (a) a $70^{\circ}$ sphere-cone at $-16^{\circ}$ angle of attack, and (b) a spherical section at $-28^{\circ}$ angle of attack. Both configurations were tested at a Mach number of 8 in high Reynolds number flow of pure $\mathrm{N}_{2}$ in the T9 tunnel of AEDC.

The turbulent flow predictions using the Baldwin-Lomax algebraic turbulence model are in good agreement with the experimental measurements. As reported in the work of Hollis, ${ }^{17}$ the experimental data have an uncertainty of $\pm 12 \%$. For the most part, predictions lie within this uncertainty band. It must be noted that computations for both shapes have not been performed with excessively fine grids. However, meshes in either case have been tailored to 
align with the bow shocks and employ sufficient grid point clustering in the wall-normal direction to ensure good resolution of the wall-bounded shear layers.

Predicted centerline distributions of pressure are shown in Figs. $6 \mathrm{a}$ and $6 \mathrm{~b}$, for $70^{\circ}$ sphere-cone and spherical section, respectively.

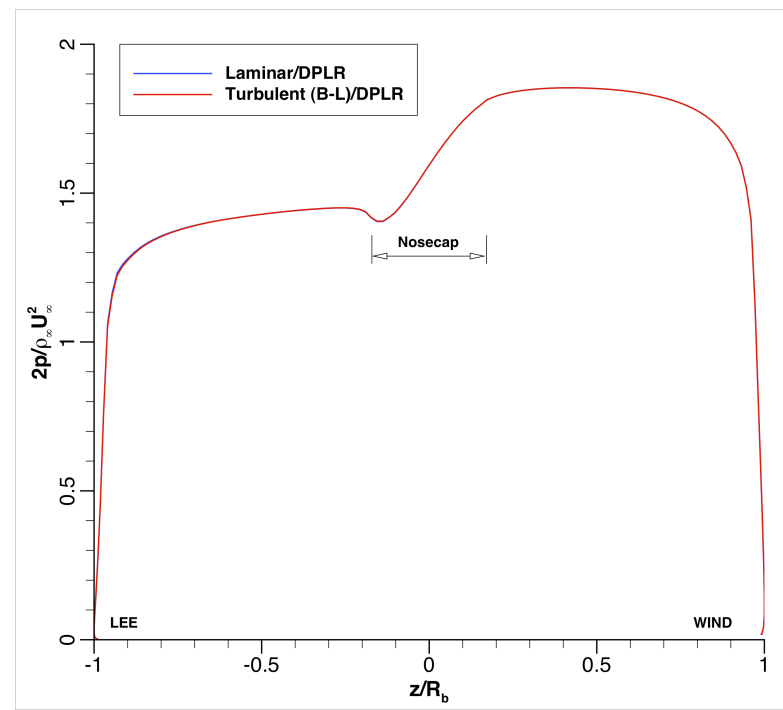

(a) $70^{\circ}$ sphere-cone, $\alpha=-16^{\circ}$

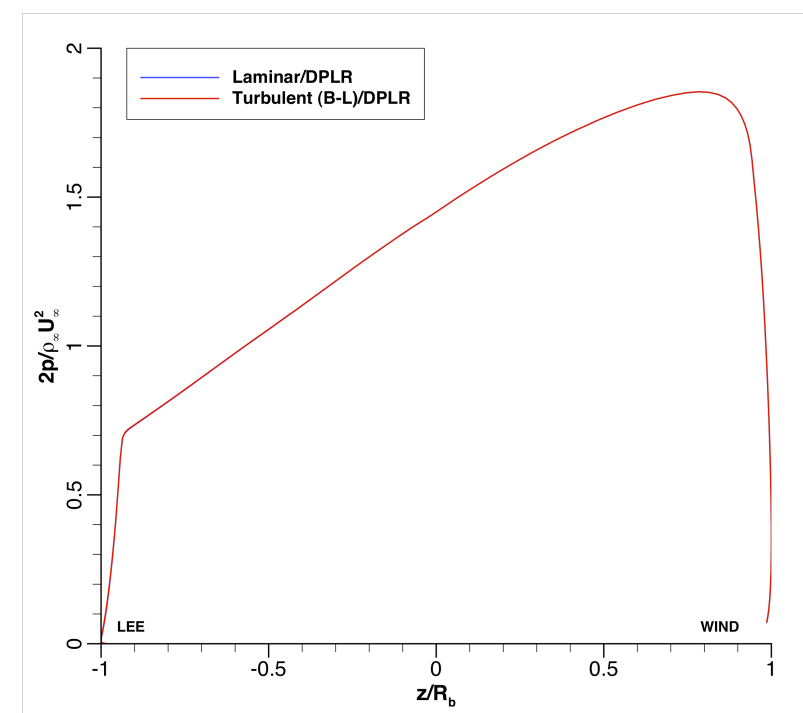

(b) Spherical section, $\alpha=\mathbf{- 2 8}{ }^{\circ}$

Figure 6. Centerline pressure distributions for: (a) a $70^{\circ}$ sphere-cone at $-16^{\circ}$ angle of attack, and (b) a spherical section at $-28^{\circ}$ angle of attack. Both configurations were tested at a Mach number of 8 in high Reynolds number flow of pure $\mathrm{N}_{2}$ in the T9 tunnel of AEDC.

In the case of the $70^{\circ}$ sphere-cone, the discontinuity in curvature (see Section IIA) at the sphere-cone junction leads to an inflection in the pressure distribution, regardless of whether the flow is laminar or turbulent. Beyond this junction, the streamwise pressure gradient is favorable (i.e., the gradient has a negative sign) towards both the windward and leeward shoulders. In contrast to this behavior, the spherical section, which does not have any curvature inflection apart from at the shoulder, has a favorable pressure gradient over the entire leeward side of the heatshield. This distribution is similar to the distribution at $-15.7^{\circ}$ for the flight case (Fig. $4 \mathrm{~b}$ ), but steeper because of the higher angle of attack $\left(-28^{\circ} v s-15.7^{\circ}\right)$. For either configuration, the pressure distributions predicted for laminar and turbulent flows are identical.

Since the T9 test case does not have any high-enthalpy effects (or real-gas effects), a second test case, that of high Reynolds number flow of dissociated $\mathrm{CO}_{2}$, is one taken from a matrix of conditions presented in the paper of Wright et al. ${ }^{18}$ The freestream condition for this case are presented in Table 5. At this condition, a $17.78 \mathrm{~cm}$ diameter $70^{\circ}$ sphere-cone ( 0.0395 -scale MSL) model made of stainless steel was tested in the T5 tunnel (a reflected shock tunnel) at Caltech. The angle of attack of the model was $-16^{\circ}$. For the freestream condition given in Table 5, the freestream Mach and unit Reynolds numbers are $M_{\infty}=4.61, R e_{\infty}=0.877 \times 10^{7} 1 / \mathrm{m}$, respectively. The unit Reynolds number for this case is about 20 times lower than in the T9 case.

Table 5. Freestream condition for a case (Shot \#2263) from the Caltech T5 Tunnel MSL test matrix.

\begin{tabular}{ccccccccc}
\hline \hline $\begin{array}{c}\boldsymbol{H}_{\mathbf{0}} \\
(\mathrm{MJ} / \mathrm{kg})\end{array}$ & $\begin{array}{c}\boldsymbol{P}_{\boldsymbol{0}} \\
(\mathrm{MPa})\end{array}$ & $\begin{array}{c}\boldsymbol{U}_{\infty} \\
(\mathrm{m} / \mathrm{s})\end{array}$ & $\begin{array}{c}\boldsymbol{\rho}_{\infty} \\
\left(\mathrm{kg} / \mathrm{m}^{3}\right)\end{array}$ & $\begin{array}{c}\boldsymbol{T}_{\infty} \\
(\mathrm{K})\end{array}$ & $\boldsymbol{c}\left[\mathbf{C O}_{2}\right]$ & $c\left[\mathbf{O}_{\mathbf{2}}\right]$ & $c[\mathbf{C O}]$ & $c[\mathbf{O}]$ \\
\hline 5.7 & 71 & 2701 & 0.17769 & 1470 & 0.898 & 0.037 & 0.065 & 0.0 \\
\hline \hline
\end{tabular}

This second case differs from the first in two ways. Firstly, the freestream is a high-temperature dissociated gas mixture consisting of $\mathrm{CO}_{2}, \mathrm{CO}$, and $\mathrm{O}_{2}$. This mixture is further heated by the bow shock that envelopes the test article. As a consequence, $\gamma$ is no longer a constant, and is a function of both temperature and chemical composition. Secondly, chemical reactions at the surface of the test article are important, even if the wall is assumed isothermal at $300 \mathrm{~K}$. Chemical reactions at the surface are recombination of atomic oxygen diffusing down to a cold surface, and oxidation of $\mathrm{CO}$. The latter is a surface-mediated reaction and often requires a noble metal such as platinum or palladium to catalyze it. 
A simple approach towards surface chemistry has been adopted in the development of environments for aerothermodynamic design (TPS material selection and sizing) of the heatshield of the MSL. The wall is assumed to be "supercatalytic" to recombination of atomic and molecular species, ${ }^{19}$ i.e., the freestream composition is completely recovered at the surface, and hence all the enthalpy in the chemical modes of the dissociated gas mixture. Since this "supercatalytic" model provides conservative estimates of surface heating, it is used for design purposes. For the case of a dissociated freestream, the term "supercatalycity" becomes ambiguous because recovery of the freestream composition at the body surface would mean that chemical enthalpy recovery is incomplete. In the work of Wright et al. ${ }^{18}$ this ambiguity was removed by assuming that the chemical composition at the body surface was $100 \% \mathrm{CO}_{2}$ regardless of the state of dissociation of the freestream. In the present work, the actual freestream composition is recovered at the surface, and it should be expected that predicted heat fluxes for this ground test would be lower than those in the work of Wright et al.

An alternate approach to the problem of surface chemistry is to assume that there are two competing rate processes for atomic oxygen adsorbed on the surface ${ }^{20}$ - one for recombination with another oxygen atom in the gas phase, and one for recombination with $\mathrm{CO}$ in the gas phase, i.e.,

(1) $\mathrm{O}+\mathrm{O}[\mathrm{s}] \rightarrow \mathrm{O}_{2}$

(2) $\mathrm{CO}+\mathrm{O}[\mathrm{s}] \rightarrow \mathrm{CO}_{2}$

This model, dubbed "fully catalytic," has had some success in replicating heating measurements made in the ballistic range. ${ }^{21}$ Heat fluxes inferred from thermal imaging of a projectile (a sphere) flying through quiescent $\mathrm{CO}_{2}$ (in the ballistic range) are in fair agreement with predictions based on the rate chemistry model. In the present work, both approaches have been used in numerical simulations of the test case, and the results are shown in Fig. 7.

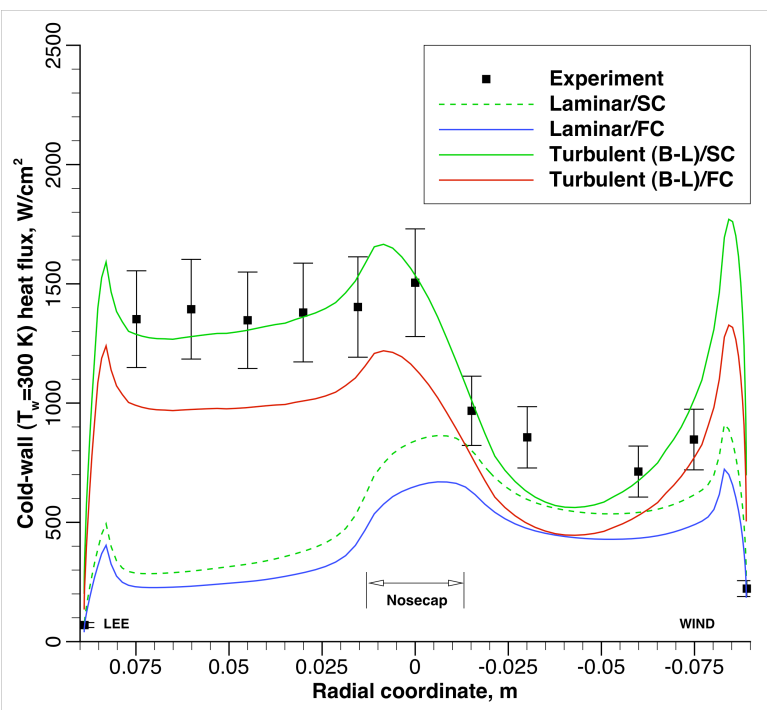

(a) Heat flux

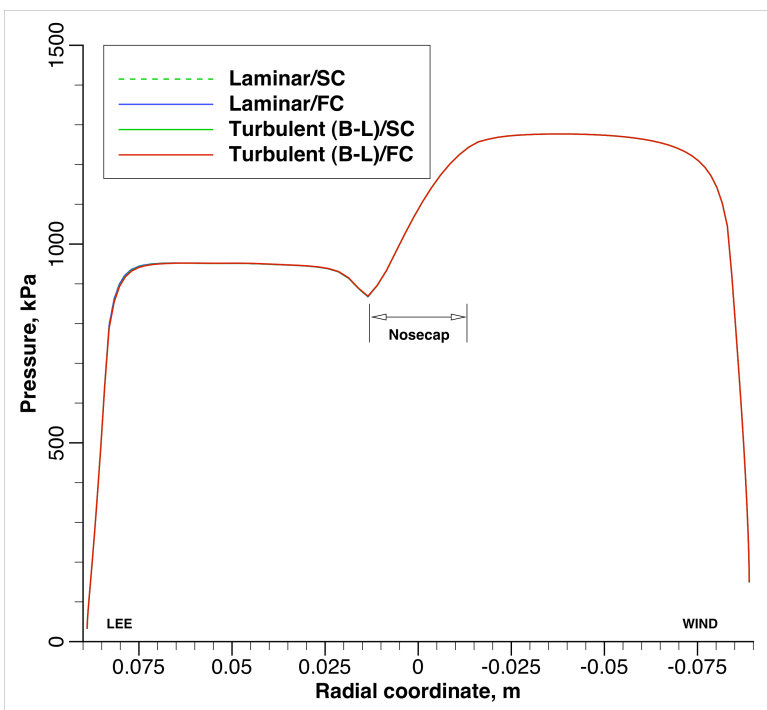

(b) Pressure

Figure 7. Centerline distributions of: (a) non-dimensional cold-wall $\left(T_{w}=300 \mathrm{~K}\right)$ heat flux, and (b) nondimensional pressure for a $17.78 \mathrm{~cm}$ diameter $70^{\circ}$ sphere-cone tested at a Mach number of 4.6 and at an angle of attack of $-16^{\circ}$ in high Reynolds flow of dissociated $\mathrm{CO}_{2}$ in the reflected shock tunnel $\mathrm{T5}$ at Caltech.

Predicted heat fluxes along the centerline of the test model are compared against measurements in Fig. 7a. An arbitrary $\pm 15 \%$ error bar is used to indicate uncertainty in measurement. The heat fluxes predicted by the "supercatalytic" wall model are in good agreement with measured data, while those predicted by the "fully catalytic" wall model are significantly lower. However, the trends in turbulent heating on the leeward flank of the cone are similar for both predictions and measurements. Although not shown in Fig. 7a, the heat fluxes predicted by the "supercatalytic" wall model employed in the present work are lower than those predicted by Wright et al. (see Fig. 15 in Ref. 18). This is as expected because of the ambiguity in the meaning of the term "supercatalytic". The variation of pressure along the centerline of the model is shown in Fig. $7 \mathrm{~b}$.

Results of numerical simulation of ground-based experiments in two different facilities make for interesting comparisons. The distributions of heat flux for the $70^{\circ}$ sphere-cone tested in T9 tunnel at AEDC (Fig. 5a) and the reflected shock tunnel in Caltech (Fig. 7a) are quite similar in that the leeward turbulent heating levels are nearly "uniform." However, the pressure distributions (Figs. 6a and 7b) show differences. Firstly, the pressure expansion at 
the sphere-cone junction is shallower for the T9 case compared to the T5 case. Secondly, while the streamwise pressure gradient is favorable toward the leeside for the T9 case, it is not favorable and neutral at best for the T5 case. Inflections in the pressure distribution and the sign of the pressure gradient are important aspects, and discussions about them are deferred until other aspects of the problems, viz., real-gas effects, angle of attack, bluntness, etc. are studied in detail.

Contours of Mach number in the pitch plane of the $70^{\circ}$ sphere-cone models tested in the T9 and T5 tunnels are shown in Fig. 8. Only three contour levels -0.01 , 1.0, and maximum freestream Mach number - are shown for each case.

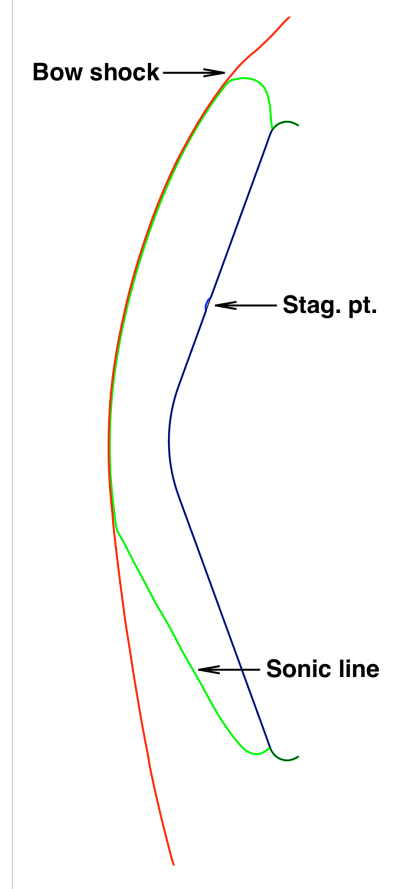

(a) T9 Model (15.24 cm diameter), $M_{\infty}=7.95$

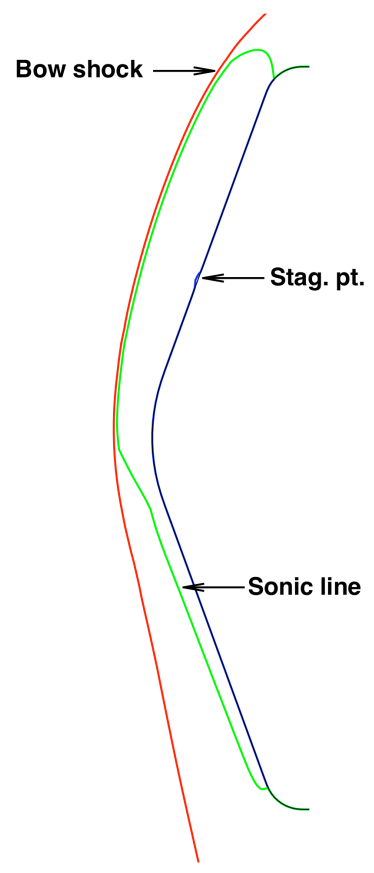

(b) T5 Model (17.78 cm diameter), $M_{\infty}=4.6$

Figure 8. Contours of Mach number in the pitch plane of the $70^{\circ}$ sphere-cone models tested in the T9 and T5 tunnels. Only three contour levels - 0.01, 1.0, and maximum freestream Mach number (7.95 or 4.6) - are shown. Both models are at $-16^{\circ}$ angle of attack.

For both the T9 and T5 models, the sonic line attaches at the shoulders (windward and leeward). However, for the T5 case, the sonic line has an inflection in the vicinity of the apex because the shock is drawn in closer to the body because of the compression of the test gas $\left(\mathrm{CO}_{2}\right)$. The behavior of the sonic line in the T5 case is still different from the flight case (Fig. 1c), where due to further compression, the sonic line attaches on the sphere-cone interface on the leeward side.

The pressure distributions from laminar and turbulent flow computations are identical to each other for both the T9 and T5 cases. This is unlike the distributions for the flight case (Fig. 4b). Could this behavior be attributed to differences in real-gas effects (catchall for excitation of internal structure of atoms and molecules and/or chemical reactions) between ground and flight? If real-gas effects are indeed the cause of the differences, then the issue of ground-to-flight traceability becomes a little more difficult to address satisfactorily.

\section{C. $70^{\circ}$ sphere-cone: Real-gas effects}

In order to study the influence of internal structure (electronic or rovibronic) of constituent atoms and molecules, and chemical reactions between them, a quick computational experiment is performed at the peak heating point of a retrograde/steep entry trajectory (see Table 3). Calculations are performed for three variations in the thermochemistry model. These models are 
Model 1: No dissociation is allowed for the $\mathrm{CO}_{2}-\mathrm{N}_{2}$ mixture. Further, internal (vibronic) structure of $\mathrm{CO}_{2}$ and $\mathrm{N}_{2}$ are not considered. Since $\mathrm{CO}_{2}$ is a linear molecule, disallowing any vibration means that the gas mixture behaves as an ideal one with $\gamma=1.4$, but with a different specific gas constant.

Model 2: Dissociation is still not allowed, but the vibrational modes of $\mathrm{CO}_{2}$ and $\mathrm{N}_{2}$ are included in the thermodynamic model. In this case, $\gamma$ is no longer a constant and depends on the temperature of the gas mixture. Thermal equilibrium is assumed, and GRC curve fits ${ }^{22}$ are employed for thermodynamic properties.

Model 3: The $\mathrm{CO}_{2}-\mathrm{N}_{2}$ mixture is allowed to dissociate behind the shock and internal structure of the constituent atoms and molecules are considered. For this particular model, the wall is assumed to be noncatalytic to recombination in order to keep the boundary condition consistent with the isothermal wall boundary condition used for the other 2 models. Additionally, thermal nonequilibrium $\left(T \neq T_{v}\right)$ is assumed in this model.

Figure 9 shows the centerline distributions of cold-wall $\left(T_{w}=400 \mathrm{~K}\right)$ heat flux and pressure obtained from laminar and turbulent flow computations for each of these three thermochemistry models.

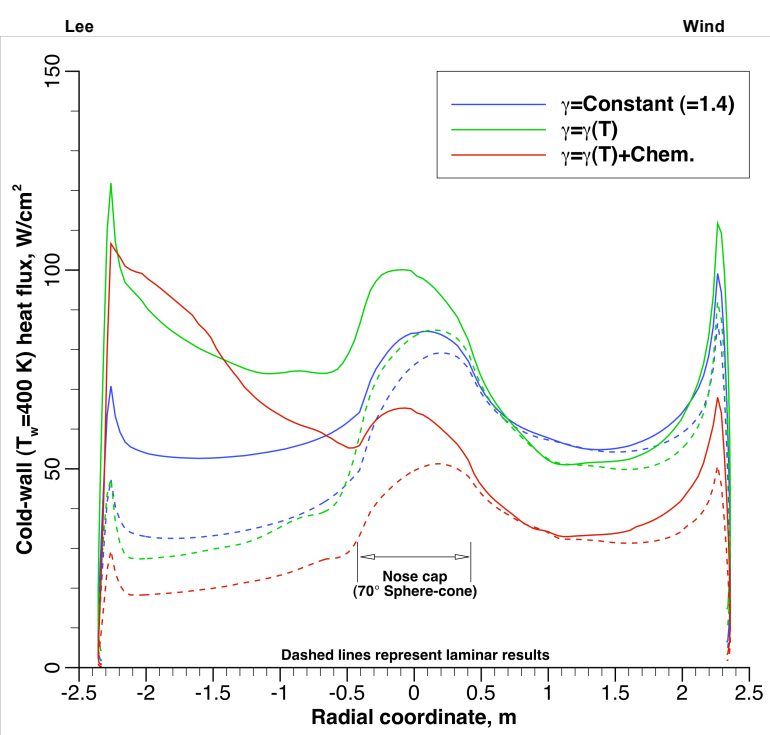

(a) Heat flux

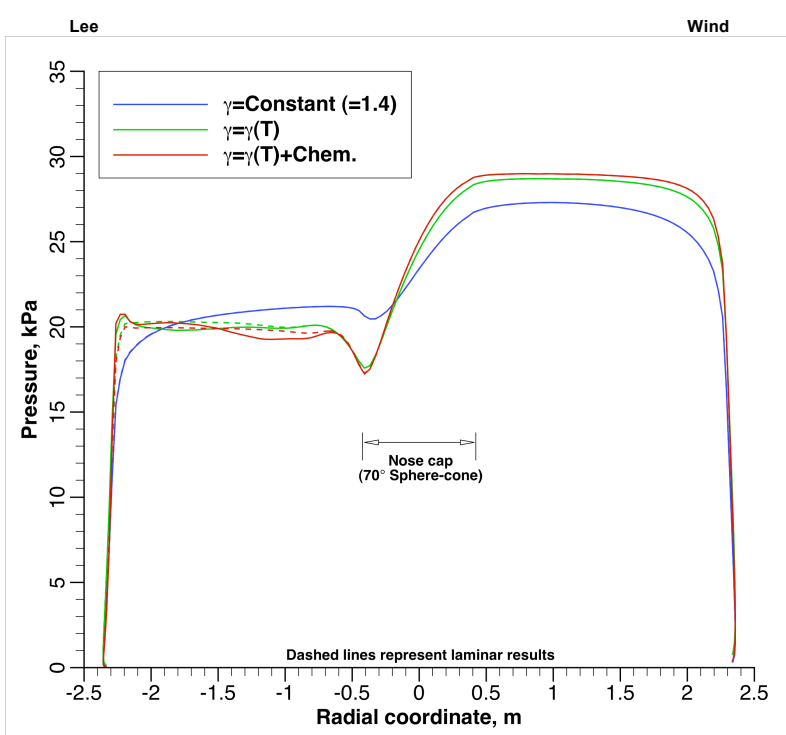

(b) Pressure

Figure 9. Centerline distributions of: (a) cold-wall $\left(T_{w}=400 \mathrm{~K}\right)$ heat flux, and (b) pressure for a $4.7 \mathrm{~m}$ diameter $70^{\circ}$ sphere-cone at a flight Mach number of 24.4 and at an angle of attack of $-15.7^{\circ}$. The freestream is a 97$3 \%$ (by mass) mixture of $\mathrm{CO}_{2}-\mathrm{N}_{2}$. Three variations in the thermochemistry model are considered: (i) without internal structure and without reactions, (ii) with internal structure and without reactions, and (iii) with both internal structure and chemical reactions. Dashed lines represent laminar results.

The distributions of laminar heat flux are more or less similar in trend for the three thermochemistry models, even if the magnitudes are different (Fig. 9a). However, the distributions of turbulent heat flux differ significantly depending on the thermochemistry model employed. An examination of the pressure distributions (Fig. 9b) makes clear the influence of internal structure and chemistry. The behavior of pressure beyond the sphere-cone junction is different for each of the three thermochemistry models that have been employed. For Model 1 ( $\gamma=$ constant), there is a small inflection in pressure, but the pressure recovers beyond the expansion and decreases towards either shoulder, i.e., the pressure gradient is favorable. Furthermore, the pressure distributions for the laminar and turbulent cases are indistinguishable for this model. For Model 2 ( $\gamma \neq$ constant, but no chemical reactions), the depth of the pressure inflection at the sphere-cone junction is larger than in Model 1. The pressure distributions for the laminar and turbulent cases, while a little different from each other, do not show secondary inflections with large amplitudes. Consequently, the rise in turbulent heating is not as dramatic. With Model 3 ( $\gamma \neq$ constant and chemical reactions are allowed to occur), the pressure distributions for laminar and turbulent flow differ, and inflections in pressure past the sphere-cone junction have larger amplitudes.

We expect that with each refinement in the model starting with Model 1, we allow the shock layer to compress more, hence drawing in the bow shock closer to the body. Contours of Mach number in the pitch plane are shown in Fig. 10. 


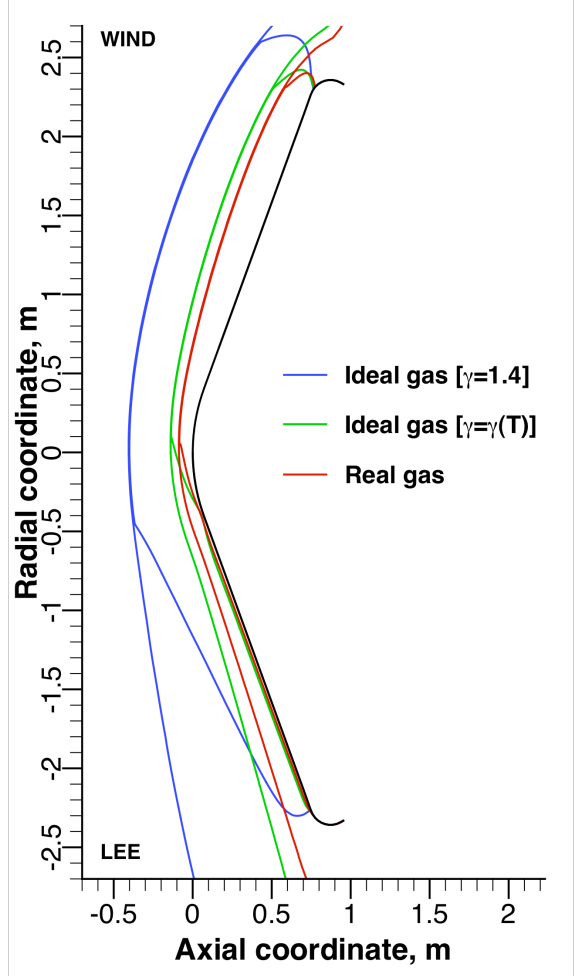

Figure 10. Pitch plane Mach number (24.4 and 1.0) contours for a $4.7 \mathrm{~m}$ diameter $70^{\circ}$ sphere-cone at a flight Mach number of 24.4 and at an angle of attack of $-15.7^{\circ}$. The freestream is a $97-3 \%$ (by mass) mixture of $\mathrm{CO}_{2}-\mathrm{N}_{2}$. Three variations in the thermochemistry model are considered: (i) without internal structure and without reactions, (ii) with internal structure and without reactions, and (iii) with both internal structure and chemical reactions.

As expected, the bow shock is drawn in closer to the body by changing the thermochemistry from an ideal-gas model $(\gamma=$ constant $)$ to a real-gas model. The sonic lines for each of these models attach at nearly the same point on the windward shoulder. However, for the $\gamma=$ constant case, the sonic line attaches on the leeward shoulder, while it attaches closer to the sphere-cone junction for the other two cases. Furthermore, the shock standoff distance decreases on the leeward side causing the bow shock to acquire a larger curvature in the vicinity of the apex of the sphere-cone. The more curved the shock is, the more likely it is to generate vorticity (Crocco's theorem ${ }^{14}$ ) and this shock-generated vorticity will likely get entrained within the wall-bounded shear layer - the notion of "entropy swallowing." Vorticity entrainment (or "entropy swallowing") is an issue for boundary-layer methods because specification of edge conditions becomes difficult in the presence of such entrainment. However, this is not an issue for Navier-Stokes equations, which are uniformly valid in the shock layer. Nevertheless, we take cognizance of the possibility of rotational flow entrainment within the wall-bounded shear layer.

We hypothesize that the behavior in pressure depends strongly on the shock-standoff distance and attachment of the sonic line on the leeward flank of the cone. A reduced shock standoff distance in the vicinity of the apex of the sphere-cone would mean increased shock curvature (the flow "feels" the presence of the nose cap), and hence generation of vorticity that will likely get entrained within the wall-bounded shear layer. A way to check this hypothesis is to blunt the cone, i.e., increase the radius, $R_{n}$, of the spherical nose cap. By blunting the cone, the shock standoff is increased, and the likelihood of vorticity getting entrained within the wall-bounded shear layer is reduced. In addition, the attachment of the sonic line on the leeward side of the cone can be studied as well.

\section{D. $\mathbf{7 0}^{\circ}$ sphere-cone: Influence of blunting}

As mentioned earlier, a rule of thumb used in the design of sphere-cone heatshields is to let the nose radius be one half the base radius, i.e., $R_{n} / R_{b}=0.5$. There does not seem to be any evidence in the extant literature that this ratio is based on any theory, but more so on the success of missions that have employed capsules based on this rule. 
Consequently, we relax this assumption and consider blunter noses for which $R_{n} / R_{b}=0.8$ and 1.1. The generatrices for these shapes are shown in Fig. 11. Also shown in the figure is the generatrix for the spherical section heatshield.

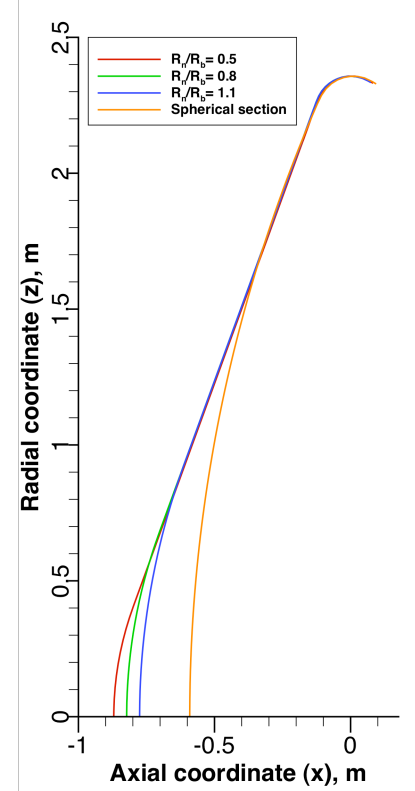

Figure 11. Generatrices for a $70^{\circ}$ sphere-cone heatshield with various values of nose bluntness, i.e., $R_{n} / R_{b}$ values. Also shown in the figure is the generatrix for a spherical-section heatshield. All profiles are lined up at the plane of maximum diameter.

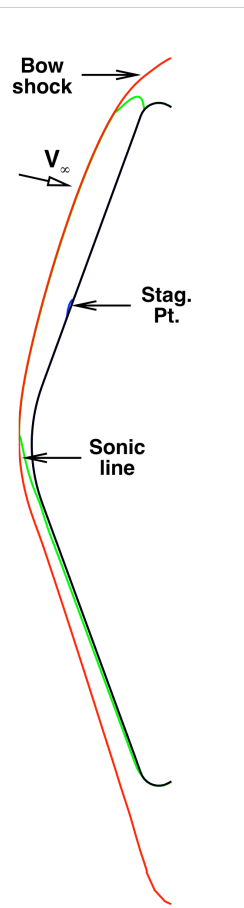

(a) $70^{\circ} \mathrm{SC}\left(R_{n} / R_{b}=0.5\right)$

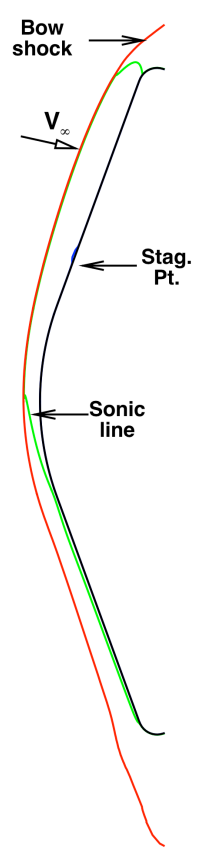

(a) $70^{\circ} \mathrm{SC}\left(\boldsymbol{R}_{n} / R_{b}=0.8\right)$

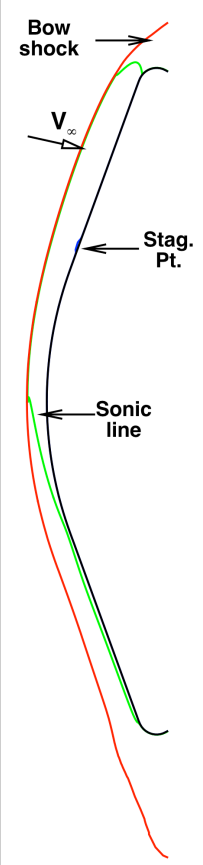

(a) $70^{\circ} \mathrm{SC}\left(R_{n} / R_{b}=1.1\right)$

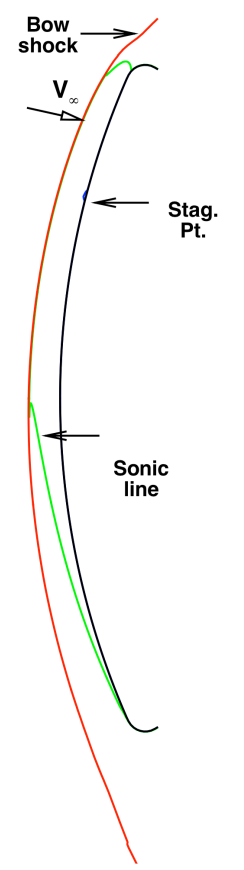

(d) Spherical-section

Figure 12. Pitch plane contours of Mach number (contour levels of $0.01,1.0$, and 24.4 are shown) for $70^{\circ}$ sphere-cone with 3 different values of nose bluntness $\left(R_{n} / R_{b}=0.5,0.8\right.$, and 1.1). Also shown are pitch plane contours of Mach number for a spherical-section heatshield of the same diameter as the $70^{\circ}$ sphere-cone. The results are from computations for a freestream velocity of $5.41 \mathrm{~km} / \mathrm{s}$ and an angle of attack of $-15.7^{\circ}$. 
It is evident that as the nose gets blunter $\left(R_{n} / R_{b}>1.1\right)$, the heatshield acquires more and more character of a spherical section. In other words, the spherical section heatshield can be viewed as the limiting case of a sphere-cone heatshield for which there is no frustum!

Both laminar and turbulent computations are performed for the configurations shown in Fig. 11 using modeling assumptions outlined in Table 2 and at the peak heating point along the retrograde/steep trajectory (Table 4). Pitch plane Mach number contours of $0.01,1.0$, and 24.4 for the sphere-cone (with different $R_{n} / R_{b}$ ratios) and the spherical section heatshields are shown in Fig. 12.

For the sphere-cone geometries, blunting the nose does indeed increase the shock standoff distance, which should translate into reduced heating on the apex of the cone, and a reduction in the curvature of the shock at the apex of the cone (Figs. 12a through 12c). Furthermore, regardless of the amount of blunting, the sonic line attaches at the windward shoulder for all cases, but appears to attach in the vicinity of the sphere-cone junction on the leeward side (Figs. 12a through 12c). Therefore, one would expect to see the inflection in pressure on the leeward side move outboard towards the shoulder with increasing nose bluntness. For the spherical section, however, the sonic line attaches at the windward and leeward shoulders (Fig. 12d).

Centerline distributions of hot-wall $(\varepsilon=0.85)$ heat flux and pressure are shown in Fig. 13.

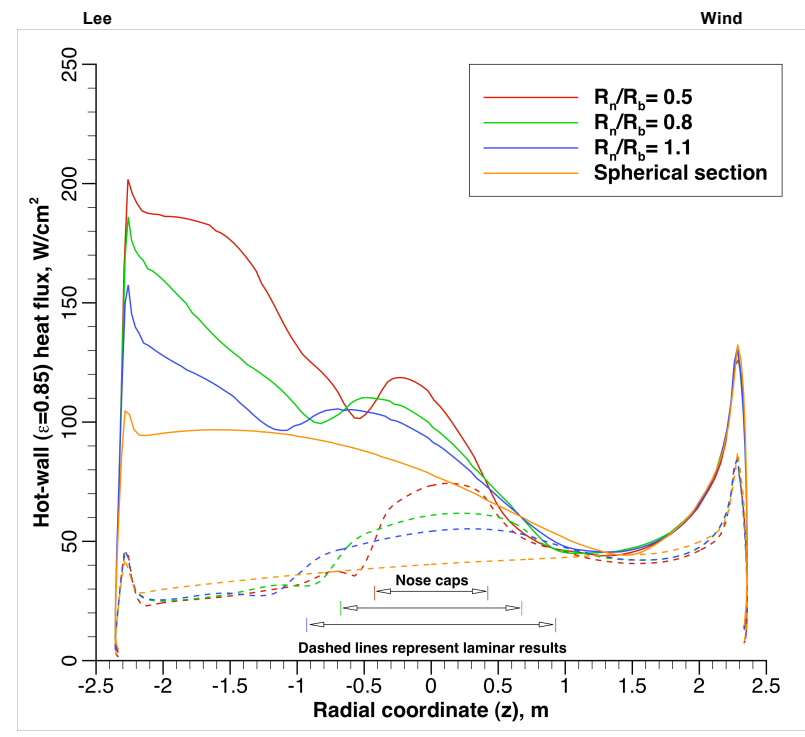

(a) Heat flux

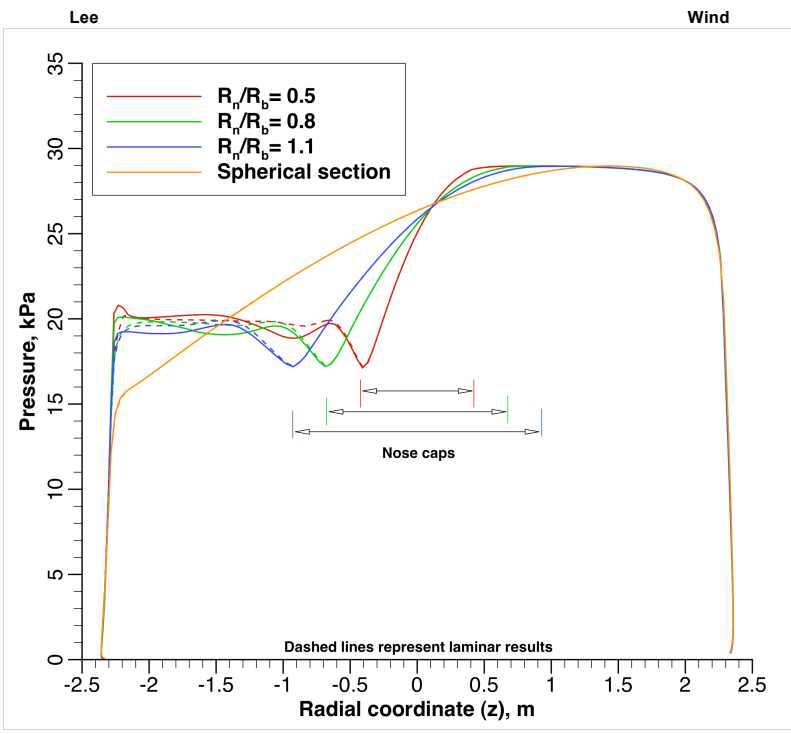

(b) Pressure

Figure 13. Laminar and turbulent centerline distributions of hot-wall $(\varepsilon=0.85)$ heat flux and pressure for a $4.7 \mathrm{~m}$ diameter $70^{\circ}$ sphere-cone at a flight Mach number of 24.4 and at an angle of attack of $-15.7^{\circ}$ for three different values of nose bluntness $-R_{n} / R_{b}=0.5,0.8,1.1$. Also shown are heat flux and pressure distributions for a spherical section at the same freestream conditions and same attitude. Dashed lines represent laminar results.

First, heating of the apex of the cone decreases with increasing bluntness, and moves more toward the heating level of the spherical section (Fig. 13a). However, despite the increased bluntness, there is enough of the conical frustum left that the laminar heating levels there are still lower than that of the spherical section heatshield. This is simply because a conical section has curvature in the circumferential direction but not in the longitudinal one. Although we have not attempted to blunt the nose any further than an $R_{n} / R_{b}$ value of 1.1 , we speculate that for values larger than 1.1, heating levels will be closer to that of the spherical section heatshield everywhere.

Next, as expected, the inflection in pressure on the leeward flank of the cone moves outboard towards the shoulder with increasing blunting of the nose (Fig. 13b). For the case of turbulent flow, pressure inflections (there are multiple points of inflection) cause rapid increase in turbulent heating. However, since there is little frustum length left with increasing nose bluntness, the "recovery" of pressure to laminar levels does not occur soon enough. Therefore, turbulent heating towards the shoulder decreases with increasing bluntness. Also, since the pressure distributions (and inflections in them) are similar for various nose radii considered, one infers that the behavior is driven by the attachment of the sonic line at the sphere-cone junction. 
All computations performed thus far have considered a fixed angle of attack of $-15.7^{\circ}$. Such an angle of attack is necessary to provide lift for the $70^{\circ}$ sphere-cone (the $L / D$ ratio for the baseline $70^{\circ}$ sphere-cone at $-15.7^{\circ}$ angle of attack is nearly 0.24 ). Such a relatively high value of $L / D$ is necessary to obtain increased downrange for the entry capsule. For larger $L / D$, one could perhaps increase the attitude of the capsule to the point where one meridian (longitude) in the pitch plane is perpendicular to the oncoming stream. For the $70^{\circ}$ sphere-cone, this angle of attack is $-20^{\circ}$. At such an angle of attack, the apex of the sphere-cone acts more like a shoulder (of large radius) and one would expect the sonic line to attach there. Increasing the angle of attack beyond this point is not particularly productive because the shoulder (small radius) and the backshell experience the oncoming flow leading to excessive heating in those areas. It is instructive to carry out a sweep in angle of attack from $0^{\circ}$ to $\theta_{\mathrm{c}}-90^{\circ}$ for the same freestream velocity and density considered until now.

\section{E. $70^{\circ}$ sphere-cone: Variation in angle of attack}

For the baseline $70^{\circ}$ sphere-cone configuration, four angles of attack $-\alpha=-5^{\circ},-10^{\circ},-15.7^{\circ}$, and $-20^{\circ}-$ are considered in this part of the study. For the freestream conditions at the peak heating point of the retrograde/steep trajectory (Table 4), laminar and turbulent computations are performed. As before, pitch plane Mach number contours (contour levels of 0.01, 1, and 24.4) are presented in Fig. 14.

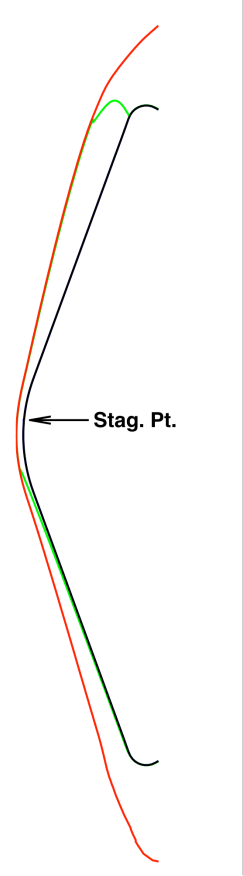

(a) $\alpha=-5^{\circ}$

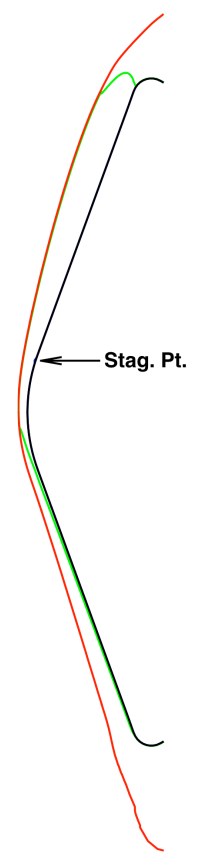

(b) $\alpha=-10^{\circ}$

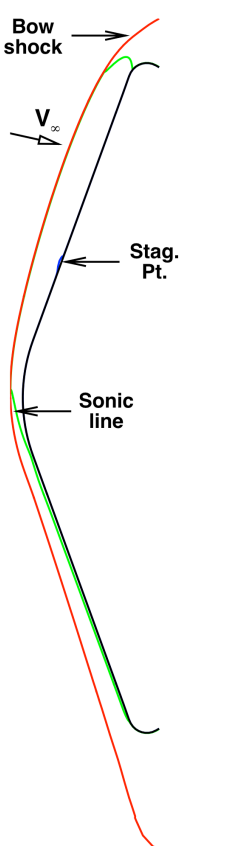

(c) $\alpha=-15.7^{\circ}$

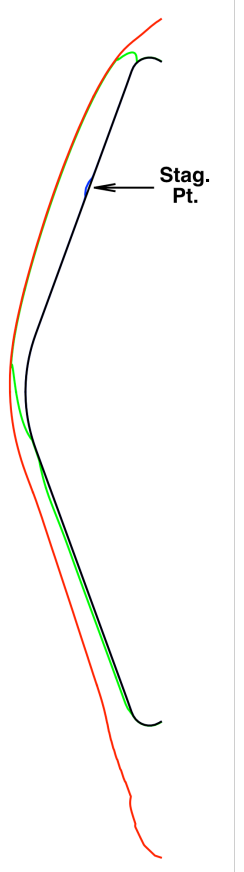

(d) $\alpha=-20^{\circ}$

Figure 14. Pitch plane contours of Mach number (contour levels of 0.01, 1.0, and 24.4 are shown) for the baseline $70^{\circ}$ sphere-cone at a freestream velocity of $5.41 \mathrm{~km} / \mathrm{s}$ and four angles of attack $-\alpha=-5^{\circ},-10^{\circ},-15.7^{\circ}$, and $\mathbf{- 2 0 ^ { \circ }}$.

First, for the lowest angle of attack $\left(-5^{\circ}\right)$, the stagnation point is on the spherical nose cap (Fig. 14a), and for the next angle of attack $\left(-10^{\circ}\right)$ is still on the sphere, but very close to the junction of the sphere and cone (Fig. 14b). For the other two angles of attack $-\alpha=-15.7^{\circ}$ and $-20^{\circ}$ - the stagnation points lie on the conical frustum (Figs. $14 \mathrm{c}$ and $14 d)$.

Next, the bow shock has the most curvature at the apex for the lowest angle of attack (Fig. 14a), with the curvature gradually decreasing with increasing angle of attack. Furthermore, the shock standoff distance progressively increases with increasing angle of attack. One would then expect heating at the apex to decrease with increasing angle of attack. For all four angles of attack, the sonic line attaches on the shoulder on the windward side, but close to the sphere-cone junction on the leeward side (Figs. 14a through 14d).

Centerline distributions of hot-wall $(\varepsilon=0.85)$ heat flux and pressure are shown in Fig. 15. 


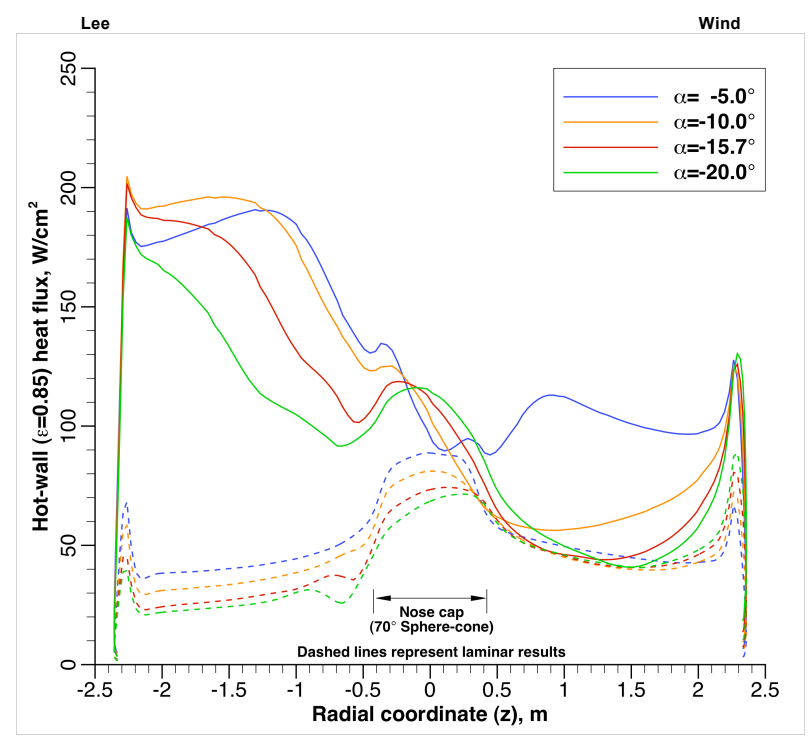

(a) Heat flux

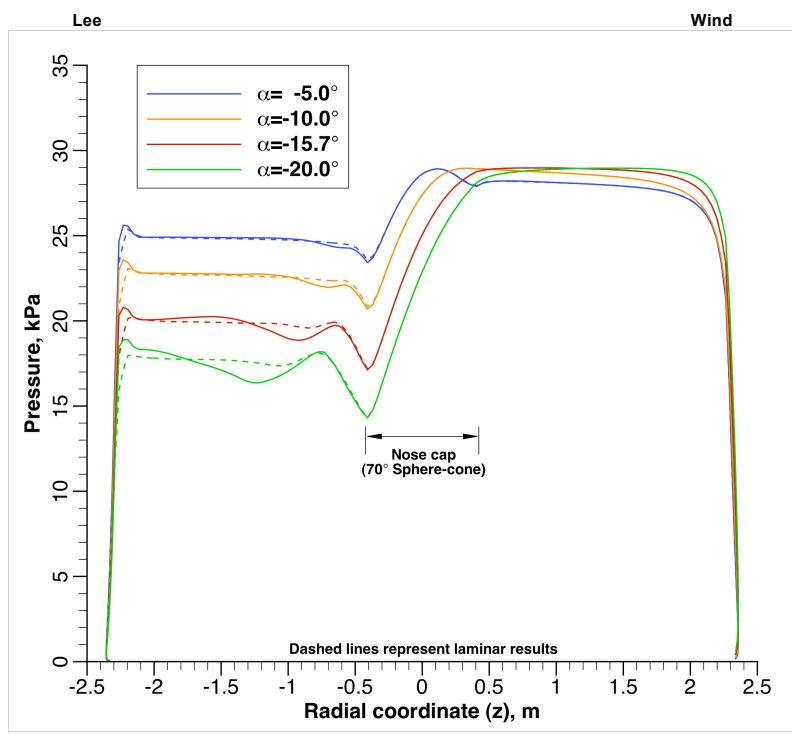

(b) Pressure

Figure 15. Centerline distributions of hot-wall $(\varepsilon=0.85)$ heat flux and pressure for a $4.7 \mathrm{~m}$ diameter $70^{\circ}$ sphere-cone at a flight Mach number of 24.4 and at angles of attack of $-5^{\circ},-10^{\circ},-15.7^{\circ}$, and $-20^{\circ}$ for one value of nose bluntness $-R_{n} / R_{b}=0.5$. Dashed lines represent laminar results.

As expected, the heating levels on the apex of the sphere-cone heatshield decrease with increasing angle of attack - the sphere-cone presents an increasingly blunt body to the flow with increasing angle of attack. At the same time, much of the leeward side is moving away from the flow, i.e., the shock standoff distance increases on the leeside with increasing angle of attack (Figs. 15a through 15d) thereby reducing the heating. At the lowest angle of attack $\left(-5^{\circ}\right)$, turbulent heating of the windward flank is substantial, and in increasing the angle of attack $(\alpha)$ from $-5^{\circ}$ to $-10^{\circ}$ there is about a $50 \%$ reduction in windward turbulent heating. Beyond this angle of attack, any decreases in windward turbulent heating are marginal because the stagnation point moves from the spherical nose cap to the conical frustum (a loss in one principal radius of curvature).

An examination of pressure distributions (Fig. 15b) shows that the differences between laminar and turbulent pressure levels increase with increasing angle of attack, although from the windside up to the sphere-cone junction on the leeward side and little beyond that, pressures obtained from laminar and turbulent flow solutions are indistinguishable from each other. Beyond the second point of inflection, there is divergence between pressures predicted using the laminar model and pressures predicted using the Baldwin-Lomax turbulence model. The most likely cause of this difference is the modification to the $A^{+}$term in the Baldwin-Lomax. This modification perhaps acts like a feedback loop especially since $A^{+}$depends on the turbulent eddy viscosity $\mu_{t}$. Furthermore, the amplitudes of pressure inflection increase with increasing angle of attack, especially at the sphere-cone junction, even for laminar flow.

A complete investigation of the $70^{\circ}$ sphere-cone requires consideration of a range of Mach numbers, dynamic pressures, and angles of attack. Since space here is limited, an attempt is made to study the impact of freestream dynamic pressure alone on the turbulent heating environment.

\section{F. $70^{\circ}$ sphere-cone: Variation in freestream conditions}

Three dynamic pressures are considered in this part of the study. For the freestream conditions at the peak heating points of three trajectories (Table 4), the dynamic pressure varies by as much as $60 \%$ between the peak heating point along the retrograde/steep trajectory and the peak heating point along the prograde/shallow trajectory. Much of this variation is due to differences in freestream density for the three cases; the freestream velocities differ by no more than $6 \%$. Laminar and turbulent computations are performed for each of three dynamic pressure conditions. Centerline distributions of hot-wall $(\varepsilon=0.85)$ heat flux and pressure are shown in Fig. 16. 


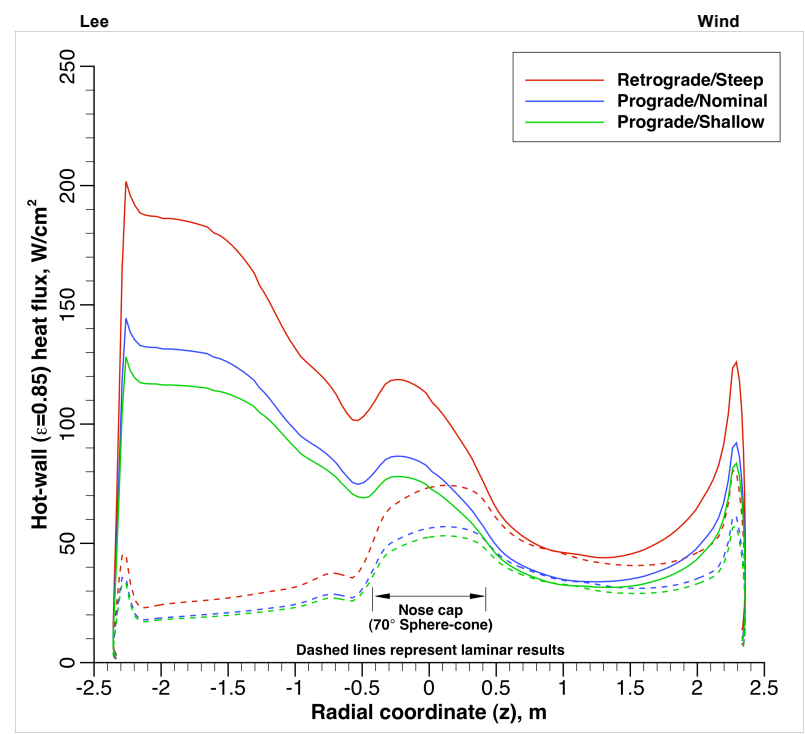

(a) Heat flux

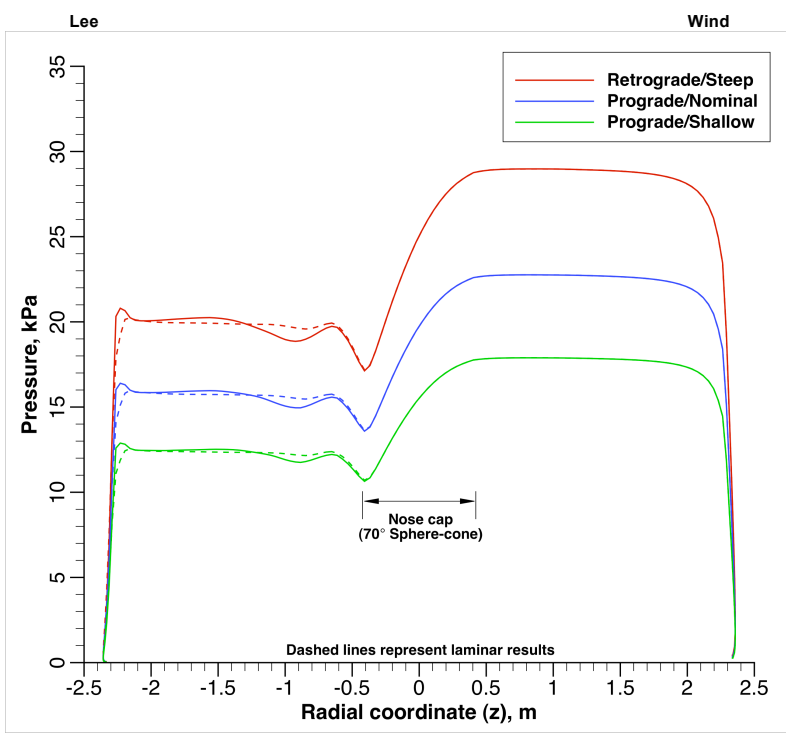

(b) Pressure

Figure 16. Centerline distributions of hot-wall $(\varepsilon=0.85)$ heat flux and pressure for a $4.7 \mathrm{~m}$ diameter $70^{\circ}$ sphere-cone at an angle of attack of $-15.7^{\circ}$ and three freestream dynamic pressure values - $14.8 \mathrm{kPa}, 11.65$ kPa, and 9.16 kPa. Dashed lines represent laminar results.

Regardless of the freestream dynamic pressure, the spatial trends in heating (laminar or turbulent) are identical the leeward side experiences a rapid rise in turbulent heating (Fig. 16a). The magnitude of heating depends on the freestream dynamic pressure - the higher the freestream dynamic pressure, the higher the heating - which is hardly surprising.

The pressure distributions (Fig. 16b) are also similar across the three freestream dynamic pressures considered. The pressures from laminar and turbulent computations are identical up to the sphere-cone junction on the leeward side. Beyond this, on the leeward flank, the pressures from turbulent predictions show multiple inflections, with the amplitudes of these inflections increasing with increasing freestream dynamic pressure.

In all of the preceding discussion, the focus has been on the $70^{\circ}$ sphere-cone shape. However, results for the alternate baseline shape - the spherical section heatshield - have been shown as well. The spherical section shape clearly has much superior aerothermal performance compared to the $70^{\circ}$ sphere-cone at the same angle of attack. Firstly, there are no inflections in surface pressure because there are no curvature discontinuities over the acreage except at the junction of the dish section and the shoulder. Secondly, the streamwise pressure gradient is always favorable, which means transition to turbulence is less likely. Thirdly, even if transition does occur on the acreage, the turbulent heating levels are much lower than those of the $70^{\circ}$ sphere-cone. Therefore, it would appear that the spherical section heatshield is a natural choice to replace the $70^{\circ}$ sphere-cone heatshield for a Mars entry capsule. However, the technology readiness level of the spherical section heatshield for flight through Mars atmosphere is low, even though it has flight heritage in Earth atmosphere. Furthermore, the aerodynamic trim characteristics for the spherical section heatshield are likely to require more ballast mass than the $70^{\circ}$ sphere-cone for a lifting entry (Viking and MSL), and compared to a $70^{\circ}$ sphere-cone, there is significant loss of packing volume for the spherical section heatshield. With the development of systems analysis frameworks such as COBRA (Co-Optimization of Blunt-body Re-entry Analysis), ${ }^{23}$ it is possible that the spherical section heatshield parameters $\left(R_{n}\right.$ and $\left.R_{s}\right)$ can be optimized to meet multiple objectives such as maximizing the drag area while keeping maximum heat flux to a minimum and reducing the amount of ballast required. However, the problem of elevating the technology readiness level of a spherical section heatshield will remain, and investments will have to be made to characterize its aerodynamics and aerothermodynamics. Since this is true of any shape, regardless of its non-Mars flight heritage, the present study explores several other options.

We seek geometries, other than the $70^{\circ}$ sphere-cone or the spherical section, that can address one or more of the issues encountered during the detailed study of the flight heritage shapes. Specifically, we seek geometries that do not have any curvature discontinuities and develop a favorable pressure gradient on the leeward side, although some may at best minimize abrupt changes in curvature as seen in traditional shapes (Fig. 3). 


\section{Geometry Modifications}

Two approaches have been taken in the present work to construct geometries with no curvature discontinuities. In the first approach, the power of a general-purpose nonlinear constrained optimization package is exploited, while still retaining the idea of a multi-segment/composite analytic generatrix. In the second approach, the use of nonuniform rational B-splines, or NURBS, which form the basis of most modern CAD software, is explored. The objective behind the use of NURBS is to replace the composite generatrix with a single segment (if possible). Based on the lessons learned from the two approaches, an attempt is also made to combine the best features of the two.

\section{A. Configurations based on nonlinear constrained optimization}

In the initial form of this approach, the heatshield geometry is still composed of three segments (like the traditional sphere-cone). The generatrix is composed of two circle segments corresponding to the nose cap and shoulder. However, instead of a straight line that is tangent to the two circles, an elliptical one is used. The nonlinear optimization package is then used with the constraints that the elliptical segment satisfies both first and second derivative continuity at either end. Details of this procedure are given in the Appendix, where it is seen that second derivative discontinuities may be minimized rather than forced to zero.

In the present study three candidate configurations, named Z, Z2, and Z3, have been constructed using nonlinear optimization. The generatrices and curvature distributions for the three configurations are shown in Fig. 17 along with the generatrices of the $70^{\circ}$ sphere-cone and the spherical section. The closed symbols shown in the figure are the lower and upper knot points, i.e., the points at which the elliptical segment is blended smoothly into the circular segments for the nose and shoulder, respectively. The coordinates of the knot points are given in Table 6. All generatrices are lined up at the plane of maximum diameter so that one can gauge any loss/gain of volume compared to the $70^{\circ}$ sphere-cone (the first baseline shape) and the spherical section (the alternate baseline shape). Visual inspection of the generatrices in Fig. 17 shows Configuration $\mathrm{Z}$ to be very similar to the $70^{\circ}$ sphere-cone, but convex everywhere. Configuration Z2 is an attempt to blunt the nose (the lower knot point is moved outboard and aft) and improve shoulder blending. Configuration Z3, developed after examining the results of Configurations $\mathrm{Z}$ and Z2, is developed as a middle ground between $\mathrm{Z}$ and $\mathrm{Z} 2$ by suitable adjustment of various constraints.

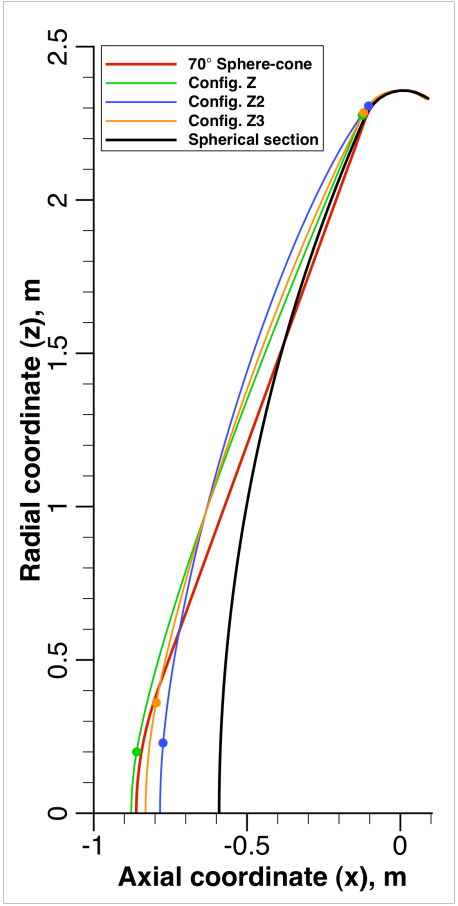

(a) Generatrices

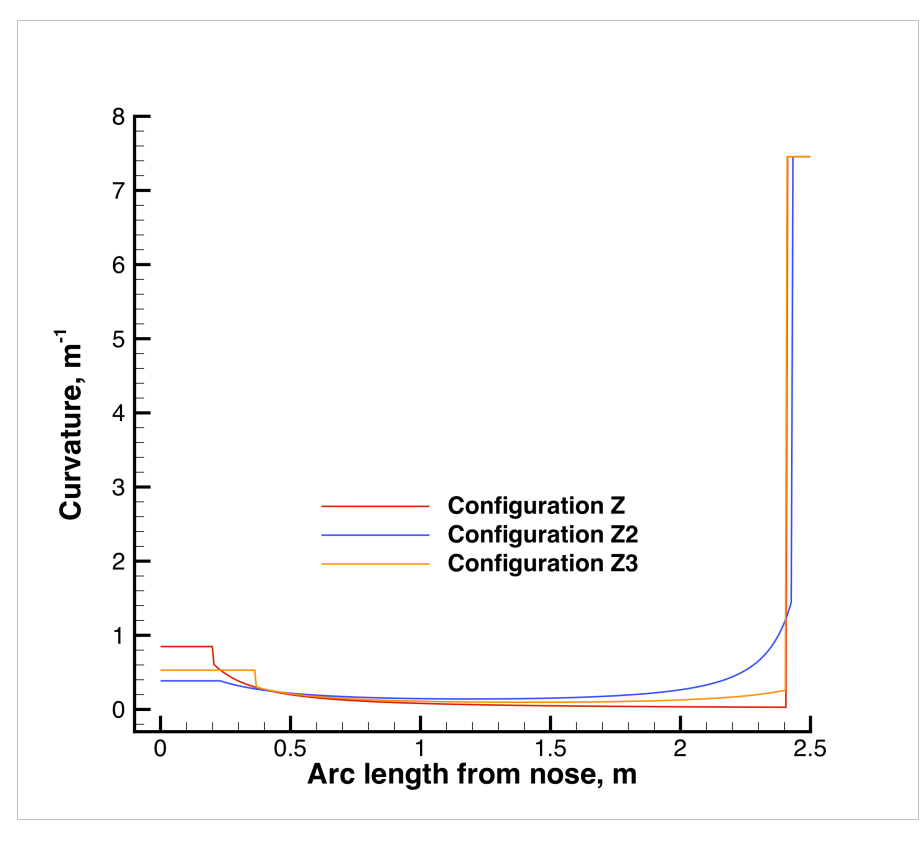

(b) Curvature distributions

Figure 17. Generatrices and curvature distributions for three alternate $4.7 \mathrm{~m}$ diameter configurations $-\mathrm{Z}$, $\mathrm{Z2}$, and $\mathrm{Z3}$ - with improved continuity in curvature at the knot points (closed symbols). The configurations are a blend of a circle, ellipse, and circle respresenting the nose cap, the "frustum", and the shoulder, respectively. Also shown are generatrices for the baseline shapes - the $70^{\circ}$ sphere-cone and the spherical section heatshield. 
Table 6. Lower and upper knot point coordinates for Configurations Z, Z2, Z3

\begin{tabular}{ccccc}
\hline \hline Configuration & \multicolumn{2}{c}{ Lower Knot Point } & \multicolumn{2}{c}{ Upper Knot Point } \\
& $\boldsymbol{x}, \mathbf{m}$ & $\boldsymbol{r}, \mathbf{m}$ & $\boldsymbol{x}, \mathbf{m}$ & $\boldsymbol{r}, \mathbf{m}$ \\
\hline $\mathbf{Z}$ & 0.017090 & 0.200000 & 0.752925 & 2.275943 \\
$\mathbf{Z 2}$ & 0.010156 & 0.229284 & 0.680203 & 2.306438 \\
$\mathbf{Z 3}$ & 0.034758 & 0.360423 & 0.711792 & 2.285000 \\
\hline \hline
\end{tabular}

The three-dimensional surfaces swept by rotating the five generatrices shown in Fig. 17 around the vehicle roll axis (the $x$ axis in the present study) are shown in profile view in Fig. 18. Configurations Z and Z3 are similar to the $70^{\circ}$ sphere-cone, while Configuration $\mathrm{Z} 2$ has more of a spherical-section character.
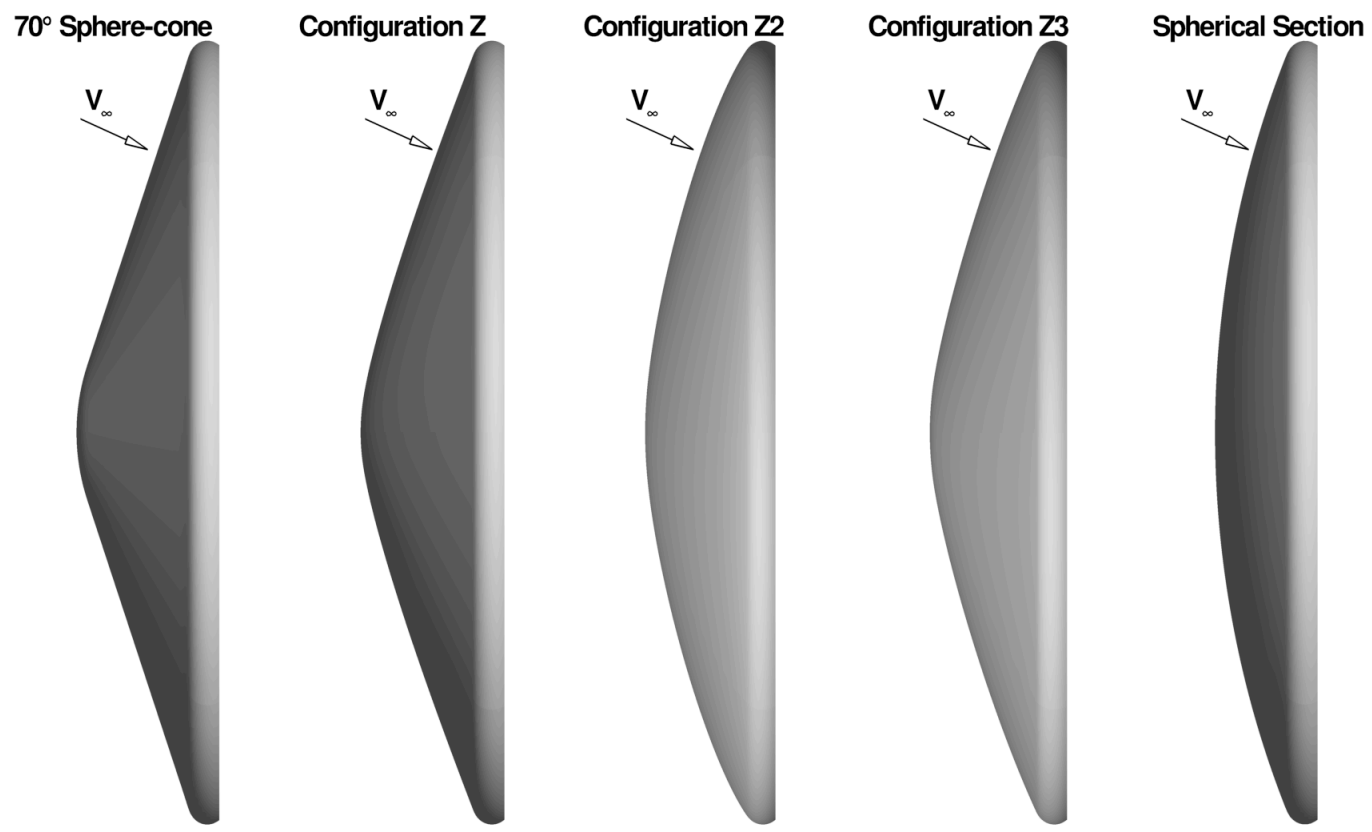

Figure 18. Three-dimensional profiles of a $70^{\circ}$ sphere-cone, Configurations $\mathrm{Z}, \mathrm{Z2}$, and $\mathrm{Z3}$ (all obtained through nonlinear optimization with constraints), and a spherical section heatshield.

Laminar and turbulent flow computations are performed at the peak heating point of the retrograde/steep entry trajectory (Table 4) with all the modeling assumptions given in Table 2. Pitch plane contours of Mach number (contour levels of $0.01,1.0$, and 24.4) for each of the three configurations - Z, Z2, and Z3 - are shown along with those for a $70^{\circ}$ sphere-cone and a spherical section in Fig. 19. 

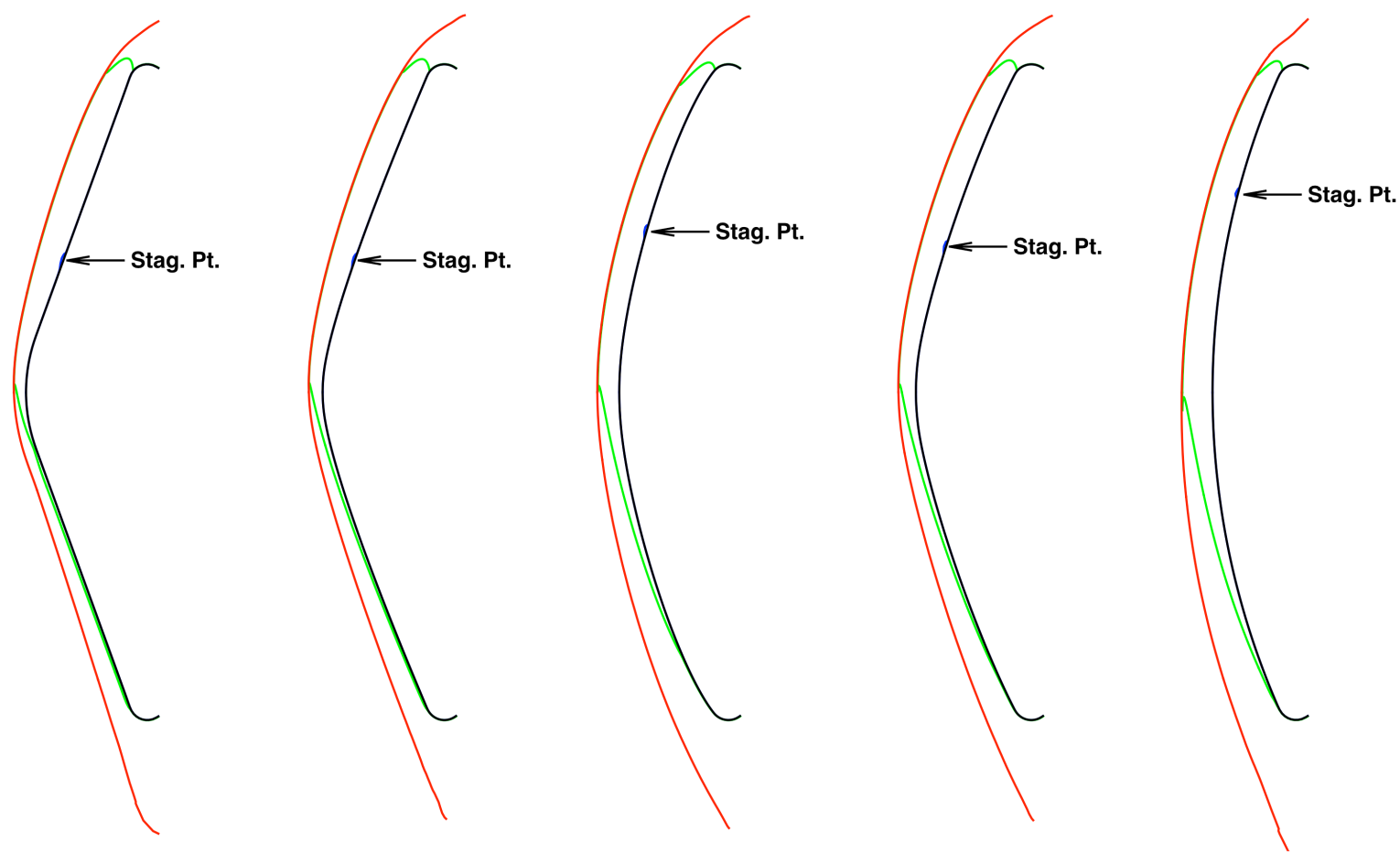

Figure 19. Pitch plane contours of Mach number (contour levels of 0.01, 1.0, and 24.4 are shown) for a $70^{\circ}$ sphere-cone, a spherical section, and three configurations (Z, Z2, and Z3) obtained using nonlinear optimization with constraints on continuity in first and second derivative between segments. Results are from computations performed at an angle of attack of $-15.7^{\circ}$ and a freestream velocity of $5.41 \mathrm{~km} / \mathrm{s}$.

It is perhaps instructive to convert the pitch plane images shown in Fig. 19 into three-dimensional representations. The sonic surface for each of the five configurations is shown in Fig. 20. The sonic bubbles for the $70^{\circ}$ sphere-cone and Configurations $\mathrm{Z}$ and Z3 are similar, while those for Configuration Z2 and the spherical section are similar. From the profiles shown in Fig. 19, one can see that Configurations Z and Z3 have a "cone like" character, and Configuration $\mathrm{Z} 2$ is closer to that of a spherical section.

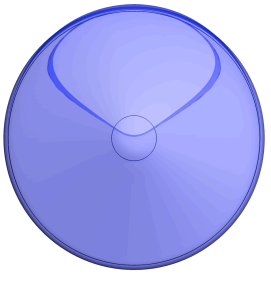

(a) $70^{\circ}$ sphere-

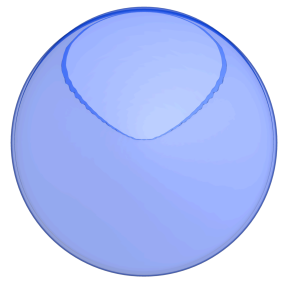

(b) Config. Z

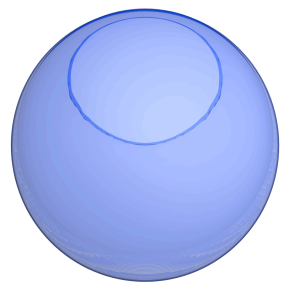

(c) Config. $\mathrm{Z2}$

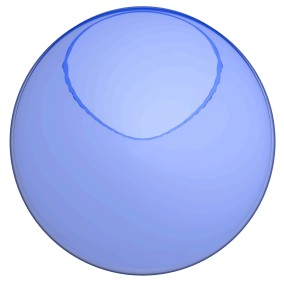

(d) Config. Z3

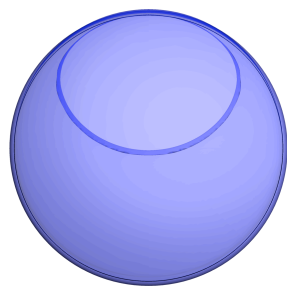

(e) Spherical section

Figure 20. Three-dimensional views of the sonic surface ( $M=1$ surface) for $70^{\circ}$ sphere-cone, a spherical section, and three configurations $(\mathrm{Z}, \mathrm{Z2}$, and $\mathrm{Z3})$ based on nonlinear optimization with constraints. Results are

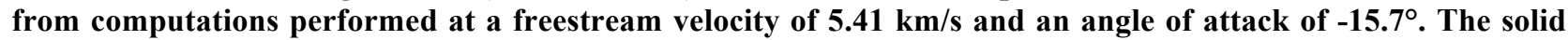
dark line at the apex of the $70^{\circ}$ sphere-cone represents the junction between the spherical nose cap and the conical frustum. 
Configuration $\mathrm{Z}$ differs little from the $70^{\circ}$ sphere-cone, especially at the shoulder. However, the modest amount of curvature added to the dish portion of the heatshield is sufficient to move the bow shock outward and prevent the sonic line from attaching close to the apex of the heatshield. Therefore, one would expect the heating on the leeward flank of Configuration $\mathrm{Z}$ to be lower than that of the $70^{\circ}$ sphere-cone, and perhaps no adverse streamwise pressure gradient in the immediate vicinity of the apex towards the leeward side. Since the apex of Configuration Z3 is a little blunter than that of Configuration $\mathrm{Z}$, a further reduction in turbulent heating on the leeward side is expected. Configuration Z2 shows characteristics of the spherical section - the dish portion has more curvature than either Configuration $\mathrm{Z}$ or $\mathrm{Z3}$, and should have the least heating of the three configurations. Furthermore, since Configuration Z2 has a more gradual blend of the dish section with the shoulder, heating at the shoulder is expected to be much less than the others. These expectations are borne out by the centerline distributions of hot-wall heat flux and pressure shown in Fig. 21.

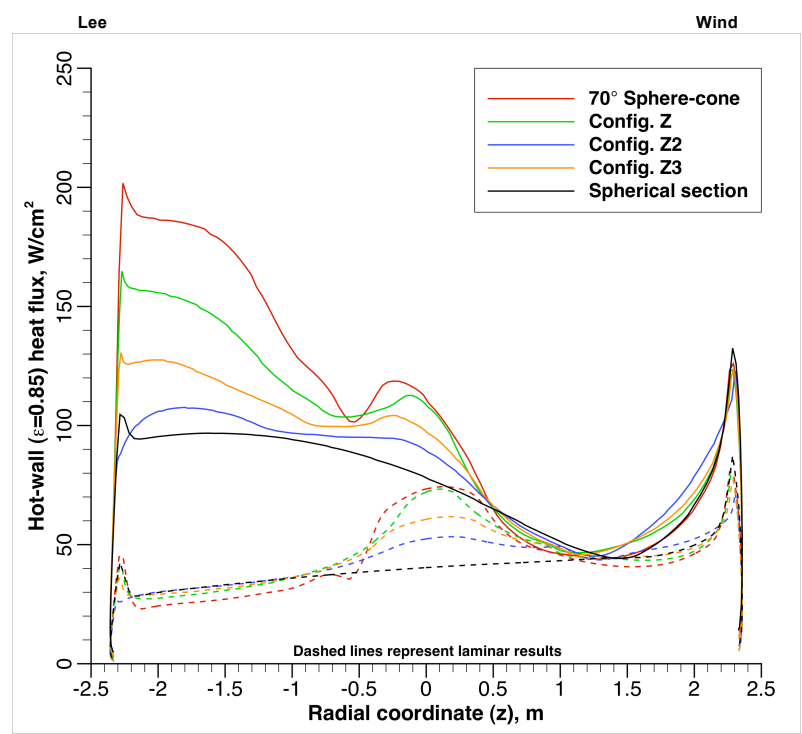

(a) Heat flux

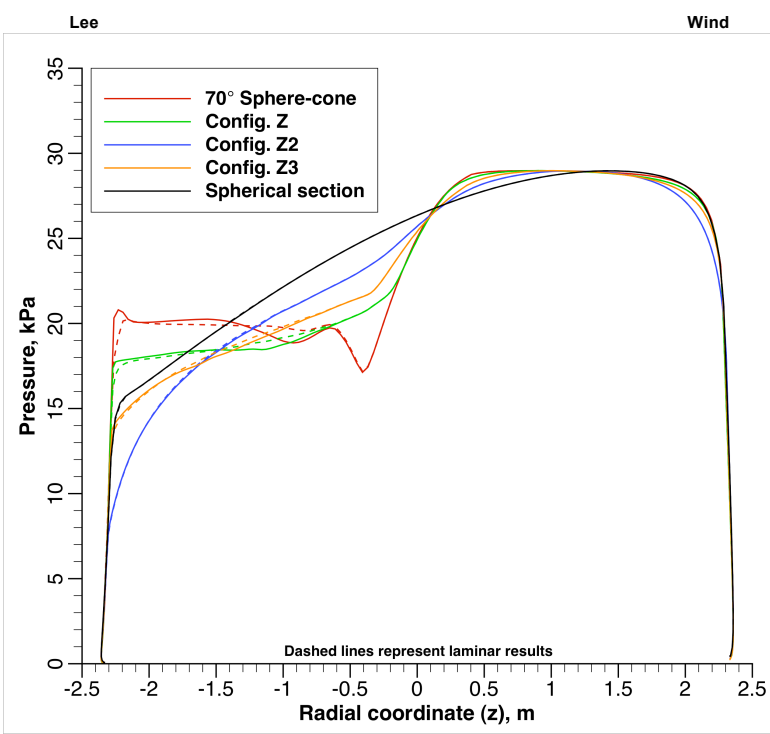

(b) Pressure

Figure 21. Centerline distributions of hot-wall $(\varepsilon=0.85)$ heat flux and pressure for $4.7 \mathrm{~m}$ diameter configurations $(Z, Z 2$, and $Z 3)$ developed using a nonlinear optimization package. These configurations are based on smooth blending between three segments - a circle, an ellipse, and a circle - corresponding to the nose cap, the dish, and the shoulder of the heatshield. Also shown are results for the $70^{\circ}$ sphere-cone and the spherical section heatshields. Dashed lines represent laminar results.

Due to the longitudinal curvature of the dish portion of the heatshield, and consequent increase in shock standoff distance, the heating of the apex decreases for Configurations Z2 and Z3 (Fig. 21a). However, since Configuration Z is very similar to the $70^{\circ}$ sphere-cone, the decrease in heating at the apex is smaller. But, the increased shock standoff distance on the leeward side helps reduce the turbulent heating level.

The pressure distributions (Fig. 21b) for the three new configurations are interesting. Unlike the $70^{\circ}$ sphere-cone case, the new blended shapes have smooth pressure distributions, and more importantly, these configurations have favorable streamwise pressure gradients over the entire wetted acreage. Favorable streamwise pressure gradients help in delaying transition, although this cannot be inferred in the present work because of the assumption of a fully developed turbulent flow from the stagnation point.

Although the heating levels are the lowest for Configuration $\mathrm{Z}$, and the streamwise pressure gradient is favorable, the pressure levels are lower than those of the spherical section heatshield. This implies that Configuration Z3 will have the lowest drag as well. Although the focus of the present paper is only on turbulent aeroheating environments and not aerodynamics, we note that the trim angle of attack for a target $L / D$ could be different for the different configurations. 


\section{B. Configurations based on NURBS}

As mentioned earlier, a second approach towards construction of surfaces without large curvature discontinuities is based on non-uniform rational B-splines or NURBS, which are the basis of most modern CAD tools. The concept of a NURBS curve is briefly explained here, and more details can be found in the work of Peigl. ${ }^{24}$

Consider the conic segment (planar curve) shown in Fig. 22. The tangents at either end point ( $A$ and $T$ ) of the segment intersect at the point $V$, and $M$ is the mid-point of the line segment connecting the points $A$ and $T$. Here $A$ can be thought of as the apex of the heatshield, and T the point of tangency of the dish and shoulder torus.

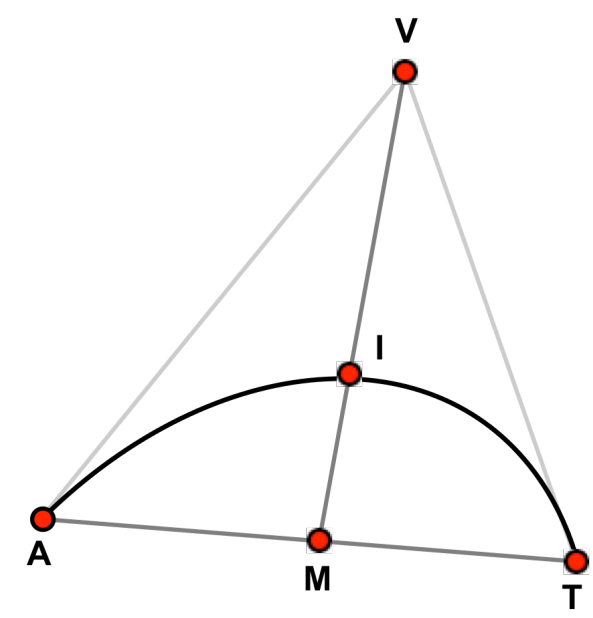

Figure 22. Construction of an elliptical segment using a non-uniform rational B-spline (NURBS) curve.

If $d(\mathrm{X}, \mathrm{Y})$ represents the distance between two points $\mathrm{X}$ and $\mathrm{Y}$ in the plane, the parameter $\rho$ of the NURBS conic is defined as:

$$
\rho=\frac{d(\mathrm{l}, \mathrm{M})}{d(\mathrm{~V}, \mathrm{M})}
$$

The parameter $\rho$ is exactly 0.5 for a parabola. For $0<\rho<0.5$ one obtains an ellipse, and for $0.5<\rho<1$ a hyperbola. We note here that the parameter $\rho$, which does not seem to have a name, is not the same as the eccentricity $\left[e=\sqrt{ }\left(1-b^{2} / a^{2}\right)\right.$, where $a$ and $b$ are, respectively, the semi-major and semi-minor axes] of an ellipse, even if the two parameters differ by exactly a factor of 2 for a parabola (i.e., $e=1$ and $\rho=0.5$ for a parabola). This aspect is evident from Eq. 7 - if $\rho \rightarrow 0$, that is $d(\mathrm{I}, \mathrm{M}) \rightarrow 0$, then $e \neq 0$, because if $e$ were zero, one would have a perfect circle. In fact, by the definition of $\rho$ given in Eq. 7, $\rho \approx 0$ is nearly a straight line. We note that it is possible to link the NURBS curve to a canonical conic via numerical means, i.e., knowing the coordinates $(x, r)$ of 5 points on the NURBS curve, the canonical equation for a general conic can be solved numerically for the various coefficients.

CFD mesh generation software programs, such as GRIDGEN, ${ }^{25}$ also provide the capability to develop simple CAD geometries using NURBS, and this capability is exercised in the present work. The intent behind the use of NURBS is to move away from circle-line-circle (or even circle-ellipse-circle) composite generatrices, and replace them with a single smooth curve, if possible. In the present study, the shoulder torus is retained, but the remainder of the heatshield is replaced by a NURBS curve.

Two new configurations - Configurations $\mathrm{X}$ and $\mathrm{Y}$ - are constructed in GRIDGEN using single elliptical segments from the apex of the heatshield to a point on the circular shoulder. The generatrices and curvature distributions for these two configurations are shown in Fig. 23 and compared with the $70^{\circ}$ sphere-cone and spherical section heatshield shapes. It should be noted that neither Configuration X nor Configuration Y has a spherical nose, and the packing volumes offered by the various shapes are different. In the present work, for Configuration $\mathrm{X}$, a value of 0.25 was chosen for $\rho$, and for Configuration Y, the value of $\rho$ was 0.3 . The angle of the tangent to the shoulder torus was different for the two configurations $-70^{\circ}$ for Configuration $\mathrm{X}$, and $66^{\circ}$ (obtained from Eq. $2 \mathrm{~b}$ with the spherical section parameters $-R_{b}=2.358, R_{n}=5.658, R_{s}=0.134$ ) for Configuration Y. 


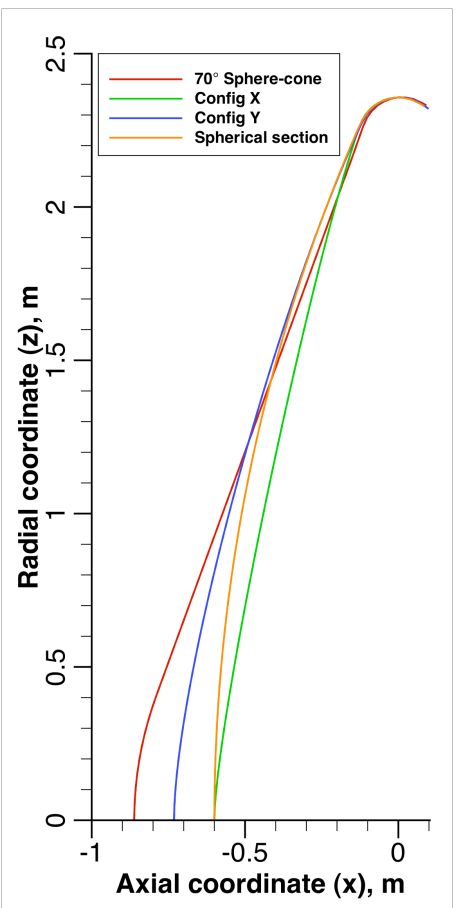

(a) Generatrices

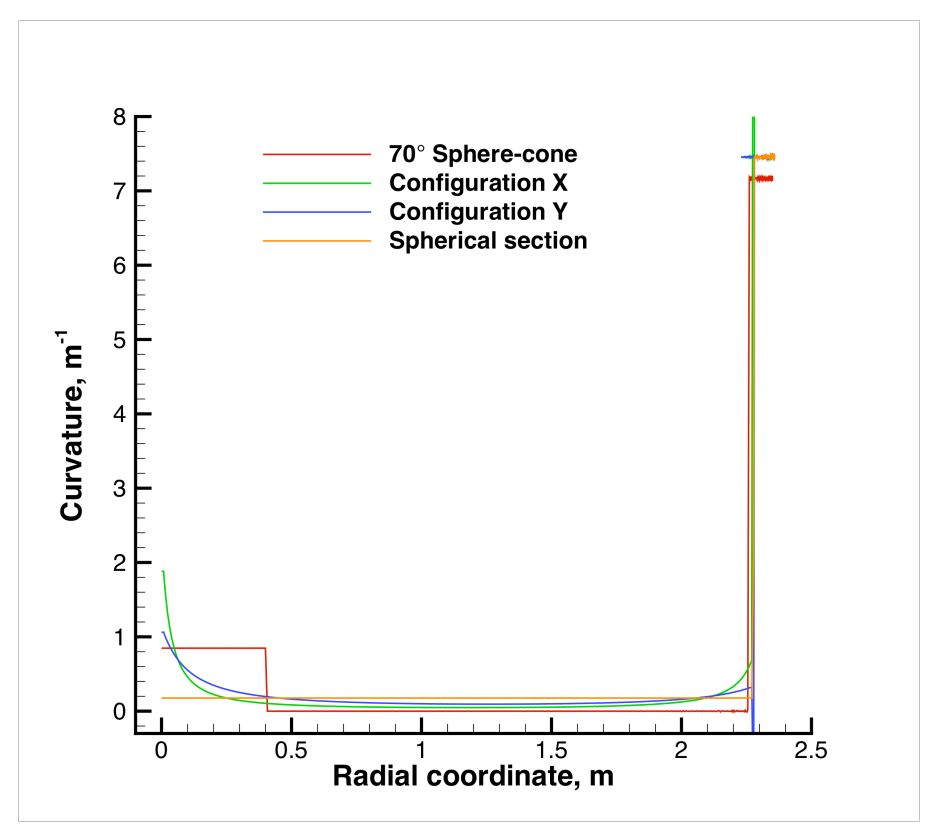

(b) Curvature distributions

Figure 23. Generatrices and curvature distributions for two alternate $4.7 \mathrm{~m}$ diameter configurations $-\mathrm{X}$ and $Y$ - based on NURBS curves. Both configurations are a blend of a NURBS ellipse for the dish with a circle (representing the shoulder). For Configuration $X$, the value of $\rho$ is 0.25 , while it is 0.3 for Configuration $Y$. The angle at which Configuration $X$ meets the shoulder torus is $70^{\circ}$, and roughly $66^{\circ}$ for Configuration $Y$ (based on the parameters, $R_{b}, R_{n}$, and $R_{s}$, of the spherical section and Eq. 2b).

Distributions of curvature (Eq. 3) for the four shapes of Fig. 23a are shown in Fig. 23b. Curvature is a constant for sections based on circles/spheres, and curvature is zero for the flat frustum section of the $70^{\circ}$ sphere-cone (the principal radius of curvature is in the circumferential direction). Although the new configurations have continuous curvature distributions except where the elliptical segments meet the circular shoulder, there are cusps at the apex of each of these geometries where the larger curvature will lead to increased heating at the apex, if the angle of attack is zero. Figure 24 shows a magnified view of the apex of a geometry constructed by rotating a tilted and displaced ellipse - the red curve is the actual generatrix, and the black curves is its mirror image about the $x$ axis. Although the radial or $r$ axis is tangential to the elliptical segment at the apex, the tilt and eccenticity of the elliptical segment control the rate of change of the second derivative, hence change in curvature.

Their smaller radius of curvature at the apex would pose a problem if capsules based on Configurations X or Y were to enter the atmosphere along a ballistic trajectory - the stagnation point would then be on the apex. However, for lifting entries this is not a particular problem because the stagnation point moves away from the apex on to the acreage, which has much less curvature. 


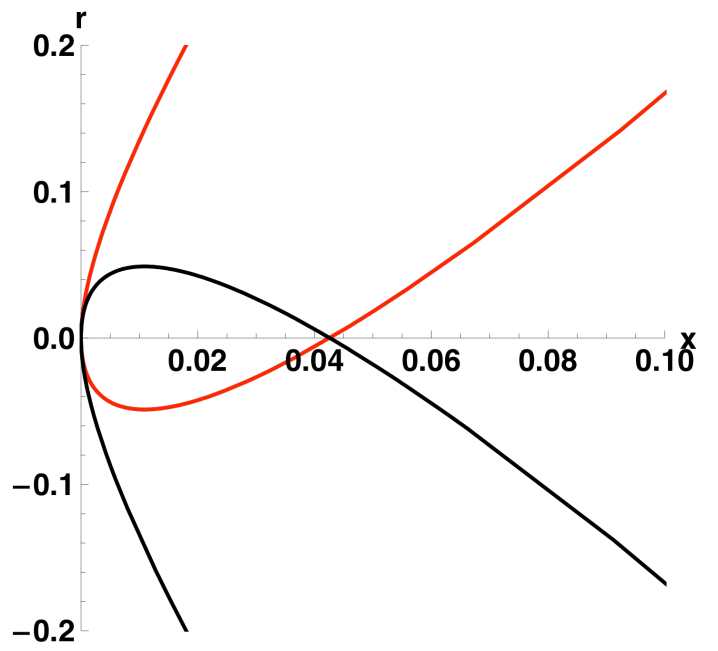

Figure 24. Magnified view of the apex of an axisymmetric configuration (Configuration $\mathrm{X}$ ) built using a displaced tilted ellipse (the red curve is the upper half and the black is its mirror image in the lower half).

The three-dimensional surfaces swept by rotating the four generatrices shown in Fig. 23a around the vehicle roll axis (the $x$ axis in the present study) are shown in profile view in Fig. 25. At the junction of the dish and the shoulder torus, the $70^{\circ}$ sphere-cone and Configuration $\mathrm{X}$ have the same slope $\left(70^{\circ}\right)$, while the spherical section and Configuration $\mathrm{Y}$ have the same slope $\left(66^{\circ}\right)$.
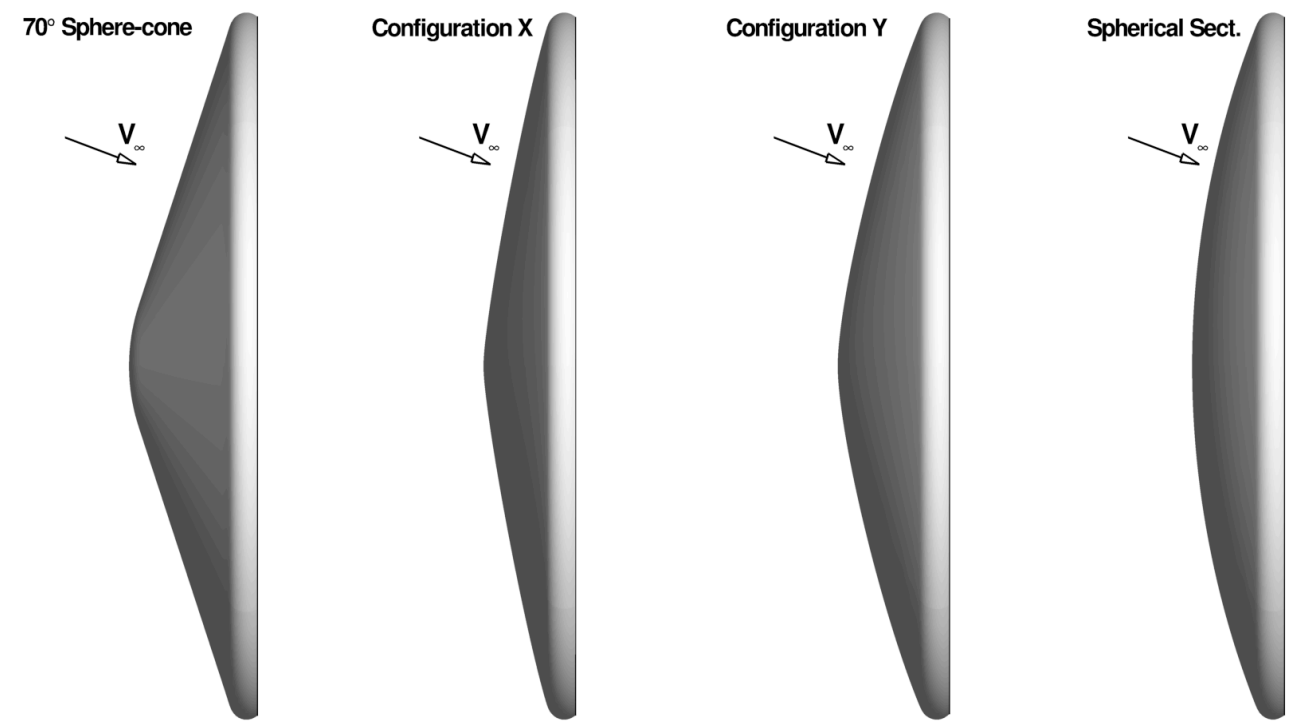

Figure 25. Three-dimensional profiles of a $70^{\circ}$ sphere-cone, Configurations $X$ and $Y$ (both NURBS-based surfaces), and a spherical section heatshield. Configuration $X$ and the $70^{\circ}$ sphere-cone have the same slope at the dish-shoulder interface, while Configuration $\mathrm{Y}$ and the spherical section have the same slope at the dishshoulder interface.

Laminar and turbulent flow computations are performed at the peak heating point of the retrograde/steep entry trajectory (Table 4) with all the modeling assumptions given in Table 2. Pitch plane contours of Mach number (contour levels of $0.01,1.0$, and 24.4) for each of the configurations - X and $\mathrm{Y}$ - are shown along with those for a $70^{\circ}$ sphere-cone and a spherical section in Fig. 26. 


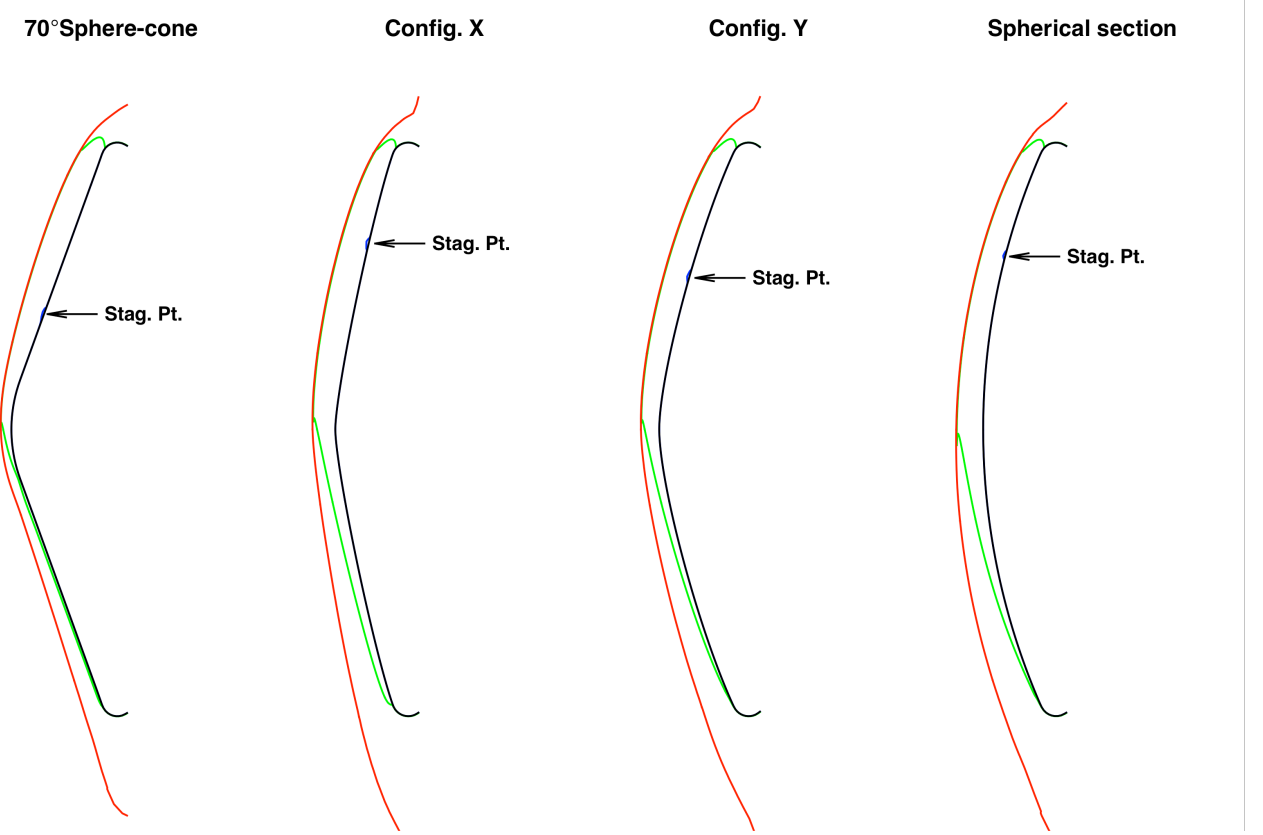

Figure 26. Pitch plane contours of Mach number (contour levels of 0.01, 1.0, and 24.4 are shown) for $70^{\circ}$ sphere-cone, a spherical section, and two configurations ( $X$ and $Y$ ) based on NURBS ellipses. Results are from computations performed at a freestream velocity of $5.41 \mathrm{~km} / \mathrm{s}$ and an angle of attack of $-15.7^{\circ}$.

Neither Configuration X nor Configuration Y has a very curved bow shock at the apex, unlike the $70^{\circ}$ spherecone, and despite the relatively sharp apices. The shock standoff distances for either configuration are larger and one expects decreased surface heating. For either configuration, the sonic line attaches closer to the leeward shoulder. Consequently, the wall-bounded shear layer will have a subsonic Mach number at the edge, and hence, reduced heating on the leeward acreage as well.

The pitch plane images shown in Fig. 26 are converted into three-dimensional representations. The sonic surface for each of the four configurations is shown in Fig. 27. It is interesting to note that while Configuration $\mathrm{X}$ and the $70^{\circ}$ sphere-cone have the same slope at the dish-shoulder junction, the sonic bubble has a greater lateral extent for Configuration X. Also, the shape of the sonic bubble of Configuration Y is similar to that of the $70^{\circ}$ sphere-cone, even though Configuration Y and the spherical section have the same slope $\left(66^{\circ}\right)$ at the dish-shoulder junction. The large lateral extent of the subsonic bubble for Configuration $\mathrm{X}$ implies a higher pressure distribution in the region compared to the other geometries. Consequently the drag performance of Configuration $\mathrm{X}$ is expected to be better. In fact, preliminary analysis of Configuration $\mathrm{X}$ using the $\mathrm{COBRA}^{23}$ framework indicated that Configuration $\mathrm{X}$ had better aerodynamic performance.

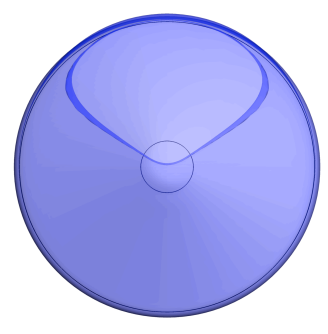

(a) $70^{\circ}$ sphere-cone

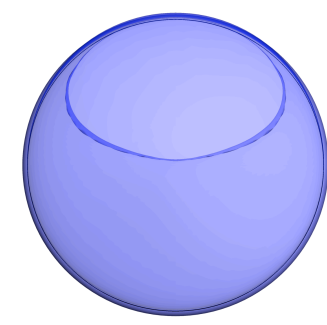

(b) Configuration $\mathrm{X}$

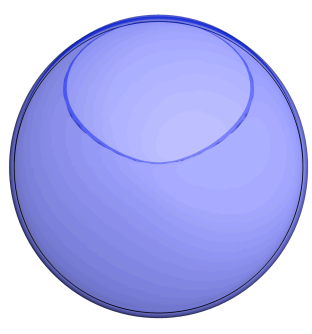

(c) Configuration $Y$

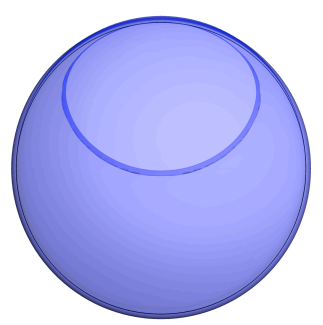

(d) Spherical section

Figure 27. Three-dimensional views of the sonic surface for $70^{\circ}$ sphere-cone, a spherical section, and two configurations ( $X$ and $Y$ ) based on NURBS ellipses. Results are from computations performed at a freestream velocity of $5.41 \mathrm{~km} / \mathrm{s}$ and an angle of attack of $-15.7^{\circ}$. The solid dark line at the apex of the $70^{\circ}$ sphere-cone represents the junction between the spherical nose cap and the conical frustum.

Centerline distributions of surface heat flux and pressure for the two configurations are shown in Fig. 28. 


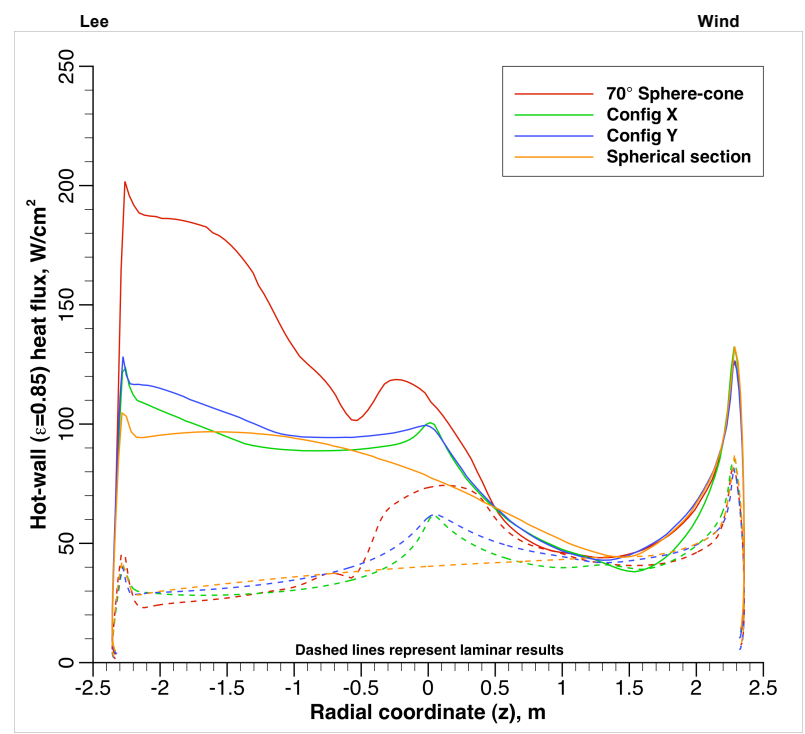

(a) Heat flux

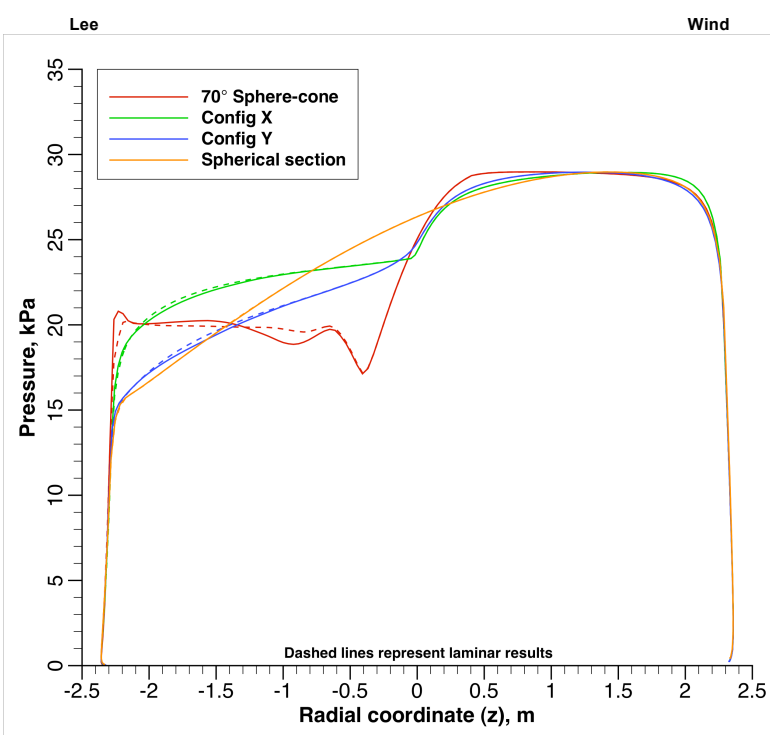

(b) Pressure

Figure 28. Centerline distributions of hot-wall $(\varepsilon=0.85)$ heat flux and pressure for $4.7 \mathrm{~m}$ diameter configurations ( $\mathrm{X}$ and $\mathrm{Y}$ ) developed using NURBS ellipses. Also shown are results for the $70^{\circ}$ sphere-cone and the spherical section heatshields. Dashed lines represent laminar results.

Barring a heating increase at the apex of the two configurations $\mathrm{X}$ and $\mathrm{Y}$, laminar heating over the acreage of the two configurations is on par with those of both the $70^{\circ}$ sphere-cone and the spherical section (Fig. 28a). Further, the heating at the apex is about $25 \%$ lower than that of the $70^{\circ}$ sphere-cone. Turbulent heating over the acreage is on par with the spherical section, and about $50 \%$ lower than that of the $70^{\circ}$ sphere-cone. The improved aerothermal performance of these new shapes over that of the $70^{\circ}$ sphere-cone can be traced to the centerline pressure distributions shown in Fig. 28b. Both Configurations X and Y exhibit an inflection in their pressure distributions at the apex. However, the streamwise pressure gradient for both configurations is favorable over the entire leeward acreage. Furthermore, the leeward pressure levels are high enough that either configuration offers better drag performance than the $70^{\circ}$ sphere-cone. For Configuration $\mathrm{X}$, the predicted centerline heat flux and pressure for three angles of attack $-\alpha=-10.7^{\circ},-15.7^{\circ}$, and $-20.7^{\circ}$ - are shown in Fig. 29.

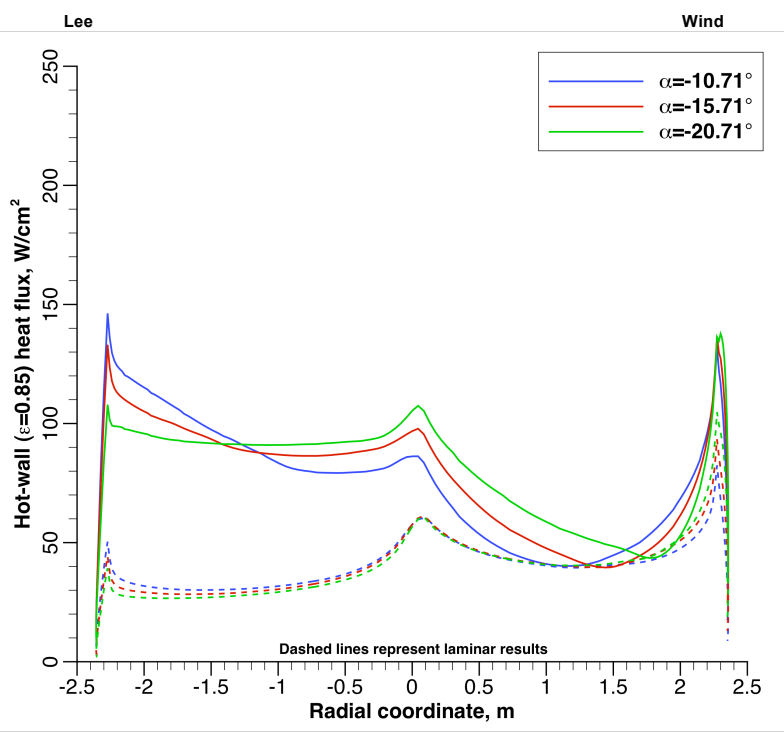

(a) Heat flux

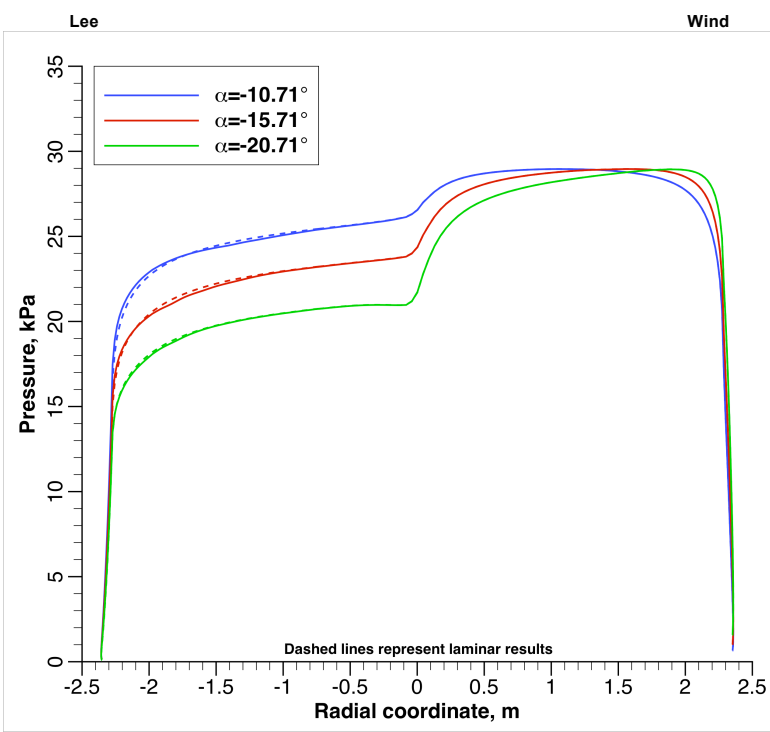

(b) Pressure

Figure 29. Centerline distributions of hot-wall $(\varepsilon=0.85)$ heat flux and pressure for the $4.7 \mathrm{~m}$ diameter Configuration $X$ at three angles of attack $-\alpha=-10.7^{\circ},-15.7^{\circ}$, and $-20.7^{\circ}$. Dashed lines represent laminar results. 
Over the relatively large angle of attack range $\left(\Delta \alpha=10^{\circ}\right)$, the aerothermal performance of Configuration $\mathrm{X}$ is still good. The turbulent heating distribution (Fig. 29a) at the highest angle of attack is reminiscent of the heating distributions seen in ground-based experiments (Figs. 5a and 7a). Across all three angles of attack considered, the pressure distribution on the leeward acreage shows a favorable pressure gradient, with the inflection in pressure at the apex of the heatshield.

\section{Curvature continuity imposed on NURBS-based profiles: Configuration X+}

Given the advantages offered by Configuration $\mathrm{X}$, an attempt is made to smoothly blend the dish section of the heatshield to a shoulder torus by applying the nonlinear constrained optimization capability to a dual-segment construction guided by the NURBS result. This should reduce the spike in heating at the leeward shoulder, and at the windward shoulder as well. A sequence of optimizations is actually performed by adjusting the constraints to approach the shape of Configuration $\mathrm{X}$ while imposing curvature continuity where the two ellipses meet (and hence everywhere, including at the shoulder). The new blended configuration (called Configuration $\mathrm{X}^{+}$) is compared with the baseline (Configuration X) in Fig. 30.

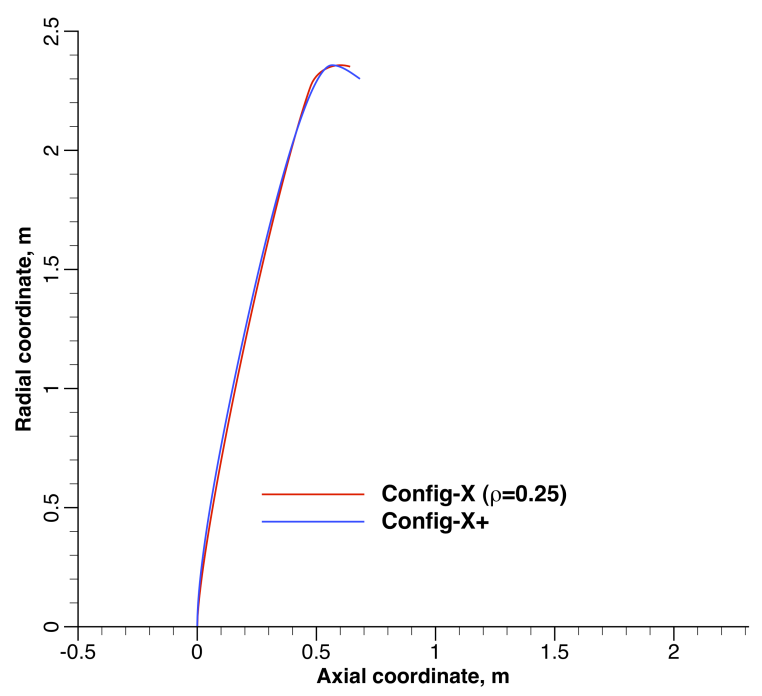

(a) Profiles

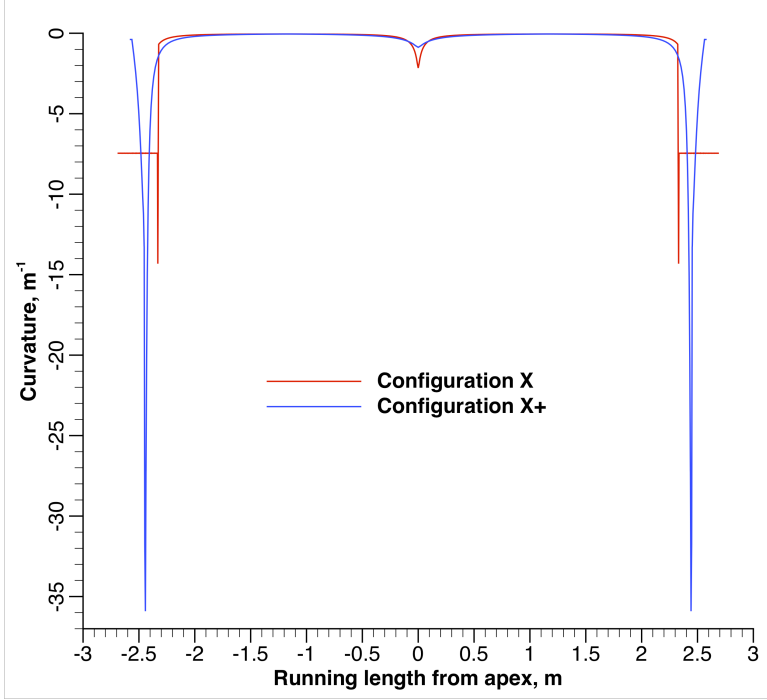

(b) Curvature distributions

Figure 30. Profiles of Configuration $X$ (elliptical section with $\rho=0.25$ and tangent to a circular torus), and Configuration $\mathrm{X}+$ (a blend of two elliptical segments - one for the dish and the other for the shoulder).

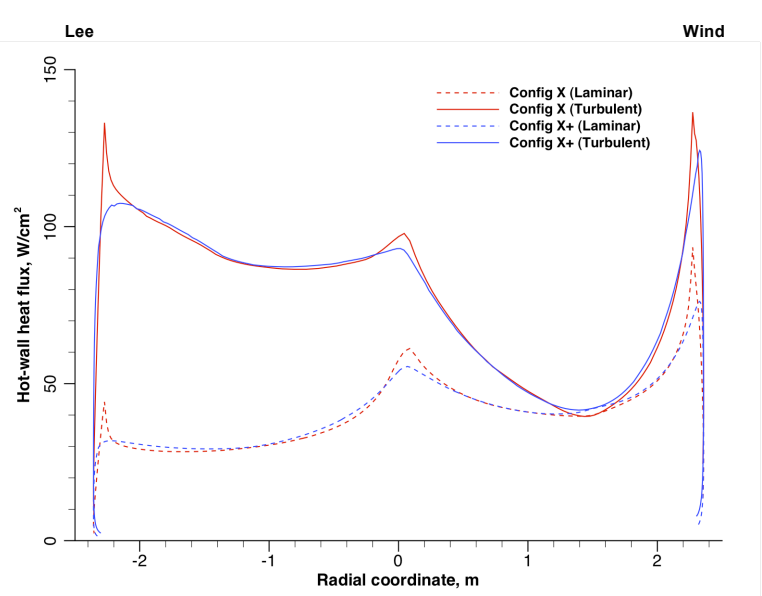

(a) Heat flux

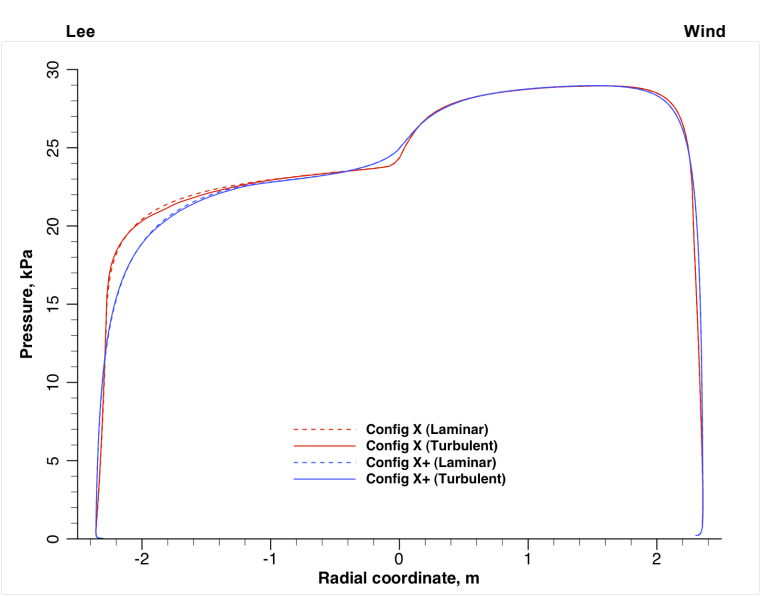

(b) Pressure

Figure 31. Comparison of centerline pressure and hot-wall heat flux distributions for Configurations $X$ and $\mathrm{X}+$. Results are from flow computations (laminar and turbulent) at a flight speed of $5.4 \mathrm{~km} / \mathrm{s}$ and $-15.7^{\circ}$ angle of attack. Dashed lines represent laminar results.

American Institute of Aeronautics and Astronautics 
The centerline distributions of pressure and hot-wall heat flux for Configurations $\mathrm{X}$ and $\mathrm{X}+$ at a flight speed of $5.4 \mathrm{~km} / \mathrm{s}$ and $-15.7^{\circ}$ angle of attack are compared in Fig. 31. While the pressures are similar on the windward side for both configurations, there is a faster decrease of pressure on the leeward side of Configuration $\mathrm{X}+$, which means Configuration $\mathrm{X}+$ will have a lower drag than Configuration X. However, the impact of blending curvature between the shoulder and dish section is clearly seen in the heating distributions, especially on the leeward shoulder Configuration $\mathrm{X}+$ has more than $25 \%$ reduction in heating on the leeward shoulder, and the distribution is much smoother. Although not as substantial, there is reduction on the windward shoulder as well.

\section{Conclusions}

Despite the rather limited focus of this study of the $70^{\circ}$ sphere-cone geometry at an angle of attack, the following broad conclusions can be drawn:

1. The discontinuity in curvature at the junction between the spherical nose and the $70^{\circ}$ conical frustum directly affects surface pressure. Pressure distributions develop an inflection at this junction, and create an adverse streamwise pressure gradient locally. Blunting the nose only serves to move the discontinuity outboard towards the leeward shoulder. Although results are not shown here in the present work, the inflection in pressure can be seen in all sphere-cone geometries, regardless of the cone angle.

2. The magnitude of the amplitude of pressure inflection at the sphere-cone junction depends directly on the proximity of the bow shock to the body, i.e., compression of the shock layer due to thermochemistry draws the shock closer to the body. If the bow shock is very close to the body, as it is in flight, the sonic line attaches at the sphere-cone junction, thus making the edge Mach number supersonic for the wall-bounded shear layer on the leeward flank of the cone. Such behavior is not observed in ground-based tests because the flow medium is either a high-Mach number (8-10) ideal gas with a limit of 6 on the density ratio across the shock, or a low-Mach number (4-6) real gas, and for either case the shock standoff distance is significantly larger than in flight.

3. For the flight cases, results of simulations assuming laminar flow show that the pressure "recovers" from the inflection at the sphere-cone junction to a nearly constant level over the conical flank on the leeward side. However, results of simulations performed with a turbulence model (Baldwin-Lomax) show that the pressure distributions have inflections beyond the sphere-cone junction, and the pressure distributions for the laminar and turbulent cases differ significantly on the leeward flank. The multiple inflections in the pressure distribution on the leeward flank of the cone do correlate with changes in the spatial gradients of heating towards the leeward shoulder.

Viable alternate shapes have been developed using two different approaches. In one approach, the concept of a multi-segment/composite heatshield is retained, i.e., the heatshield is assumed composed of a spherical nose cap and shoulder torus. However, in a departure from the $70^{\circ}$ sphere-cone geometry, which joins the nose and shoulder with a conical frustrum, these alternate shapes are joined by a "cone-like" surface but with two principal radii of curvature (the regular right circular conical frustum has only one principal radius of curvature). The power of nonlinear constrained optimization is used to blend the various segments better by reducing (if not eliminating) curvature discontinuities between the segments. The configurations developed in this approach seem to fix most of the issues with the $70^{\circ}$ sphere-cone, and show superior aerothermal performance.

Viable alternate shapes have also been developed using NURBS (non-uniform rational B-spline) curves, with the express intent of moving away from multi-segment/composite heatshields. Configurations developed using this approach also fix issues with the $70^{\circ}$ sphere-cone and show superior aerothermal performance. Constrained optimization was also applied to capture the essentials of a NURBS-based result while replacing the shoulder circle with an ellipse to achieve curvature continuity everywhere.

No attempt has been made, nor a claim, that the newly developed shapes have "optimal" performance when considering volume and packaging, although a one-off computation using the Co-Optimization of Blunt-body Reentry Analysis (COBRA) ${ }^{23}$ framework developed at NASA Ames Research Center indicated that Configuration X was close to a Pareto optimal front. ${ }^{26}$

In closing we note that claims about the new shapes, even if "optimized" using sophisticated techniques/methods, are all based on simulations, and that there is no substitute for a focused experimental program to understand the aerostability characteristics (especially dynamic stability characteristics) of these shapes. 


\section{Appendix: Blending Conics Via Constrained Optimization}

While traditional sphere-cone generatrices are constructed algebraically, and families of more refined shapes can be developed with CAD and grid generation packages such as GRIDGEN, a convenient alternative is to exploit the power of a general-purpose nonlinear constrained optimization package. If highly converged solutions are obtained, any geometric constraints (such as matching of first and second derivatives at blend points) are typically satisfied to virtually full machine precision. This is comparable to solving sets of nonlinear equations but easier to implement. More importantly, some quantity of interest (an objective function) can also be minimized or maximized in the process, so the results are optimal in some sense-invariably with at least one of the constraints active (at a bound).

The variables being optimized are typically coordinates of blend points and coefficients of curves such as conics. Equality constraints (such as for ensuring a blend point lies on some curve, or for matching slopes at blend points) are simply coded as expressions involving the variables, or the difference between two such expressions, with the associated upper and lower bounds specified as zero. Inequality constraints (such as those on conic coefficients that ensure the conic is an ellipse) are similarly coded as expressions, with one of the bounds typically specified as slightly above or below zero.

A good gradient-based optimization method has the option to compute partial derivatives of the objective and constraint functions via forward or central differencing, so the application program does not need to provide any gradient information. In a reusable software framework, the application specifics are confined to the routines that read the variables and constraints by name, read the choice of objective function, evaluate the objective and constraint functions, and write results in plottable form.

\section{Configuration Z Construction Details}

Details of the implementation that led to the Configuration $\mathrm{Z}$ generatrix are presented as illustration of the power and convenience of the constrained optimization approach.

Blend points $\mathrm{N}$ and $\mathrm{S}$ on the nose and shoulder circles respectively are permitted to slide along the circles that are part of the generatrix of the underlying sphere-cone, taken to have a $70^{\circ}$ half angle in this case. Radial coordinate $r$ is synonymous with $y$. A tilted ellipse is substituted for the conical flank, adding convexity and some volume. Initially, specifying a flank height (maximum distance from the ellipse to the straight line of the sphere-cone) was envisaged as a parameter defining a family of shapes. However, preserving the slope continuity at $\mathrm{N}$ and $\mathrm{S}$ (let alone curvature) proved to be so restrictive that this height was not specified in practice. Instead, bounds on the coordinates of $\mathrm{N}$ and $\mathrm{S}$ were specified so as to preserve reasonable portions of the circles, and the blending was made as good as possible by minimizing the [sum of squares] mismatches in second derivatives at $\mathrm{N}$ and $\mathrm{S}$.

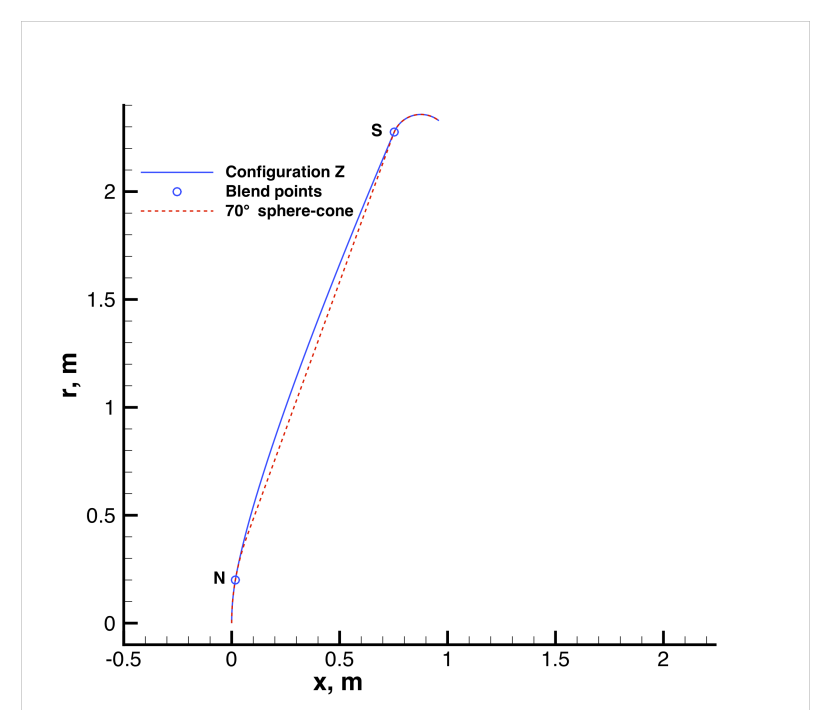

Figure A1. Configuration $\mathrm{Z}$ construction.

The input control file shown below shows how nine optimization variables are specified as coordinates $\left(x_{N}, y_{N}\right)$, $\left(x_{S}, y_{S}\right)$ and the coefficients of the flank ellipse $x^{2}+2 h x y+b y^{2}+2 g x+2 f y+c=0$. The conditions for this to be an ellipse and not a parabola or hyperbola are $b-h^{2}>0$ and $b c-f^{2}-h(h c-f g)+g(h f-b g)<0$. The "RHO" inputs are multipliers on possible contributions to the objective function, and only the mismatches in $d^{2} y / d x^{2}$ were employed for Configuration Z. No linear constraints entered the picture, and the eight nonlinear constraints serve to make $\mathrm{N}$ lie on both the nose circle and the flank curve (with analogous constraints on $\mathrm{S}$ ), to make that curve an ellipse, and to force first derivative continuity at $\mathrm{N}$ and S. Miscellaneous controls specify the underlying sphere-cone to a capsule forebody module, followed by namelist-type inputs to the optimization package NPOPT. ${ }^{27}$ The two variable bounds and seven out of eight constraint bounds that were active at the Configuration $\mathrm{Z}$ solution are bolded in the solution summary that follows the input control file below. 
OPTIMIZE Control File (Circle-Ellipse-Circle Blending)

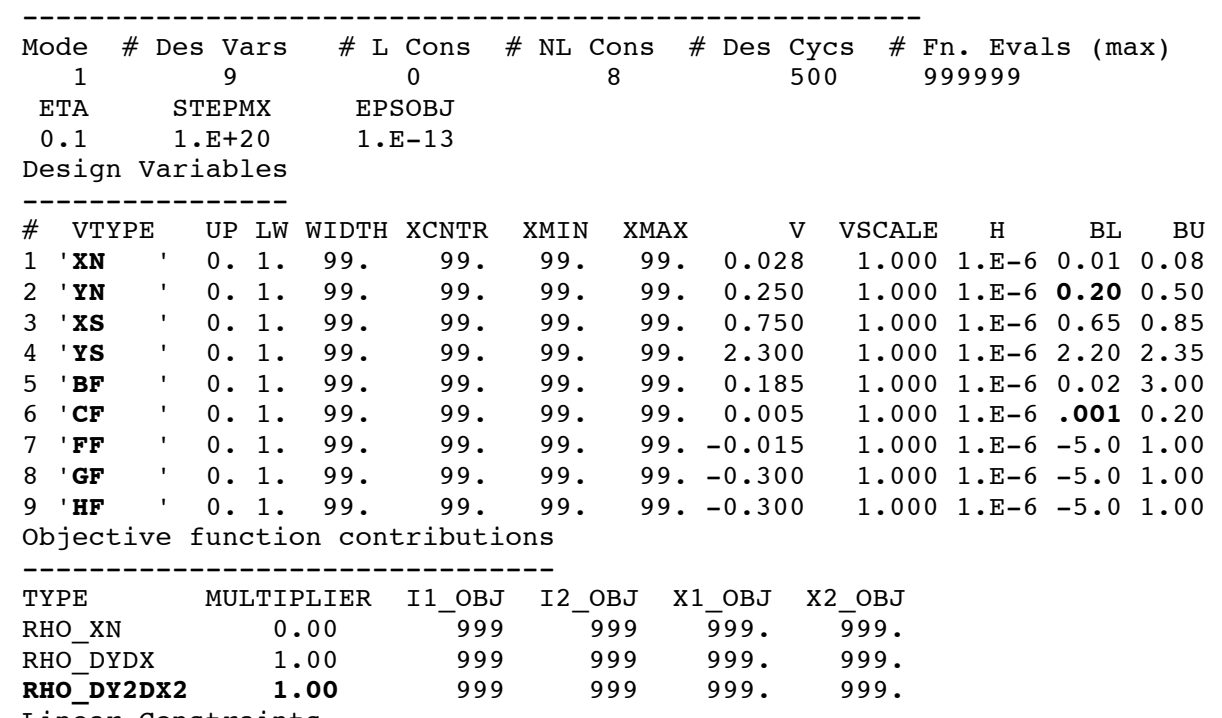

Linear Constraints

J LCTYPE BOUNDLO BOUNDUP TLCON ILCON

Nonlinear Constraints

\begin{tabular}{|c|c|c|c|c|c|c|c|}
\hline $\mathrm{J}$ & NLCTYPE & BOUNDLO & BOUNDUP & XNLCON & INLCON & JNLCON & SNLCON \\
\hline 1 & 'RESID_NN' & 0.000 & 0.000 & 999. & 999 & 999 & 10.0 \\
\hline 2 & 'RESID_NF' & 0.000 & 0.000 & 999. & 999 & 999 & 10.0 \\
\hline 3 & 'RESID_SF' & 0.000 & 0.000 & 999. & 999 & 999 & 10.0 \\
\hline 4 & 'RESID_SS' & 0.000 & 0.000 & 999. & 999 & 999 & 10.0 \\
\hline 5 & ' ELLIPSE E 1 ' & 0.003 & 9999.0 & 999. & 999 & 999 & 10.0 \\
\hline 6 & ' ELLIPSE2 ' & -9999.0 & -0.003 & 999. & 999 & 999 & 10.0 \\
\hline 7 & 'DERIV1_N' & 0.000 & 0.000 & 999. & 999 & 999 & 1.0 \\
\hline 8 & 'DERIV1_S' & 0.000 & 0.000 & 999. & 999 & 999 & 0.1 \\
\hline
\end{tabular}

Miscellaneous Controls: Underlying sphere-cone definition

1.178787 ! RADIUS_NOSE

2.357574 ! RADIUS_BASE

0.1341402 ! RADIUS SHOULDER

70. ! HALF_CONE_ANGLE

35. ! SKIRT ANGLE

0.006675 ! SKIRT_LENGTH

401 ! \# discretization points, approximately uniform

\$NPOPTIONS

LEVELVER $=1$, MAJORPL $=10$, MINORPL $=0$, MINORIL $=1000$, NPRFREQ $=0$,

LEVELDER $=0$, DIFFERENCE_INTERVAL $=-1 . \mathrm{E}-6$, PENPARAM $=0 ., \mathrm{STEPLIM}=0.01$,

TOLLIN $=1 . \mathrm{E}-6$, TOLNLIN $=1 . \mathrm{E}-9$, TOLOPT $=1 . \mathrm{E}-7$,

\$END

\section{Output Summary}

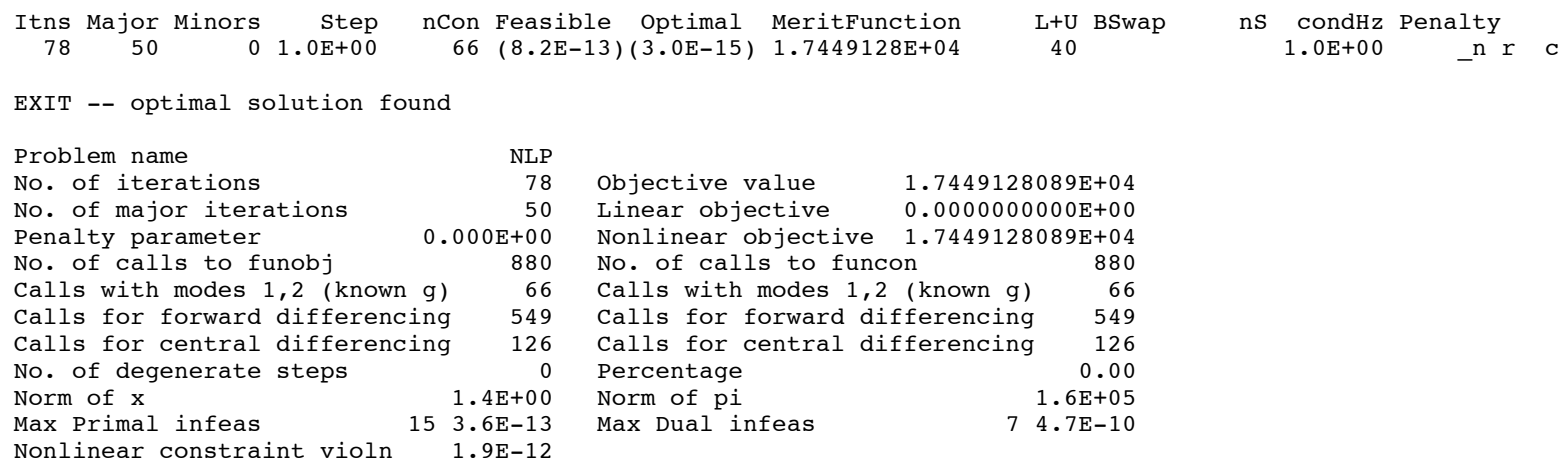

American Institute of Aeronautics and Astronautics 


\begin{tabular}{|c|c|c|c|c|c|c|c|}
\hline \multicolumn{2}{|c|}{ Variable } & State & Value & Lower bound & Upper bound & Lagr multiplier & Slack \\
\hline variable & 1 & FR & $0.1709049 \mathrm{E}-01$ & $0.1000000 \mathrm{E}-01$ & $0.8000000 \mathrm{E}-01$ & . & $0.7090 \mathrm{E}-02$ \\
\hline variable & 2 & LL & 0.2000000 & 0.2000000 & 0.5000000 & 61680.84 & • \\
\hline variable & 3 & FR & 0.7529250 & 0.6500000 & 0.8500000 & • & $0.9707 \mathrm{E}-01$ \\
\hline variable & 4 & FR & 2.275943 & 2.200000 & 2.350000 & - & $0.7406 \mathrm{E}-01$ \\
\hline variable & 5 & FR & 0.2440739 & $0.2000000 \mathrm{E}-01$ & 3.000000 & . & 0.2241 \\
\hline variable & 6 & LL & $0.1000000 \mathrm{E}-02$ & $0.1000000 \mathrm{E}-02$ & 0.2000000 & 856998.1 & • \\
\hline variable & 7 & FR & $-0.1195725 \mathrm{E}-01$ & -5.000000 & 1.000000 & . & 1.012 \\
\hline variable & 8 & FR & $-0.8743908 \mathrm{E}-01$ & -5.000000 & 1.000000 & - & 1.087 \\
\hline variable & 9 & FR & -0.4802948 & -5.000000 & 1.000000 & - & 1.480 \\
\hline \multicolumn{2}{|c|}{ Nonlin constrnt } & State & Value & Lower bound & Upper bound & Lagr multiplier & Slack \\
\hline nlncon & 1 & LL & $-0.6938894 E-14$ & . & . & -3211.126 & $0.6939 \mathrm{E}-14$ \\
\hline nlncon & 2 & LL & $-0.2498002 \mathrm{E}-14$ & - & - & -3456.580 & $0.2498 \mathrm{E}-14$ \\
\hline nlncon & 3 & LL & $-0.1931788 \mathrm{E}-11$ & - & - & -8913.383 & $0.1932 \mathrm{E}-11$ \\
\hline nlncon & 4 & LL & $-0.1804112 \mathrm{E}-14$ & • & • & -11049.88 & $0.1804 \mathrm{E}-14$ \\
\hline nlncon & 5 & FR & 0.1339079 & $0.3000000 \mathrm{E}-01$ & 99990.00 & • & 0.1039 \\
\hline nlncon & 6 & UL & $-0.3000000 \mathrm{E}-01$ & -99990.00 & $-0.3000000 \mathrm{E}-01$ & -65221.14 & $-0.3609 E-12$ \\
\hline nlncon & 7 & LL & $-0.1882938 \mathrm{E}-12$ & • & • & 12989.36 & $0.1883 \mathrm{E}-12$ \\
\hline nlncon & 8 & LL & $-0.7278622 \mathrm{E}-13$ & - & - & 333845.9 & $0.7279 \mathrm{E}-13$ \\
\hline
\end{tabular}

DESIGN VARIABLES AT DESIGN ITERATION 65

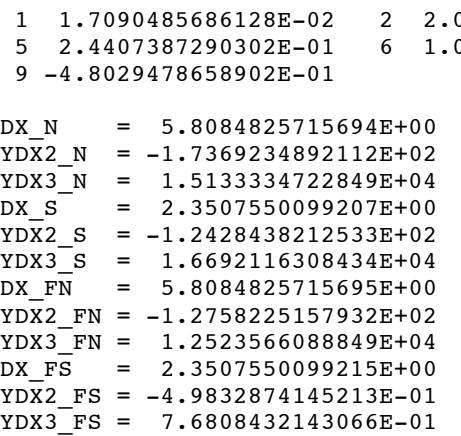

Contributions to the objective:

$\begin{array}{ll}\text { RHO_XN } & 0.000000000000000 \mathrm{E}+00 \\ \text { RHO_DYDX } & 5.652379684678066 \mathrm{E}-25 \\ \text { RHO_DY2DX2 } & 1.744912808922693 \mathrm{E}+04\end{array}$

RHO_DY2DX2 1.744912808922693E+04

NLCON: Nonlinear constraint check:

\begin{tabular}{|c|c|c|c|c|}
\hline \# & NLCTYPE & INLCON & XNLCON & CURRENT VALUE \\
\hline 1 & RESID NN & 999 & 999.0000 & 0.000000 \\
\hline 2 & RESID_NF & 999 & 999.0000 & 0.000000 \\
\hline 3 & RESID $\mathrm{SF}$ & 999 & 999.0000 & 0.000000 \\
\hline 4 & RESID SS & 999 & 999.0000 & 0.000000 \\
\hline & ELLIP $\bar{S} E 1$ & 999 & 999.0000 & 013391 \\
\hline & ELLIPSE2 & 999 & 999.0000 & -0.003000 \\
\hline & DERIV1 N & 999 & 999.0000 & 0.000000 \\
\hline & DERIV1 $\mathrm{S}$ & 999 & 999.0000 & 0.000000 \\
\hline
\end{tabular}
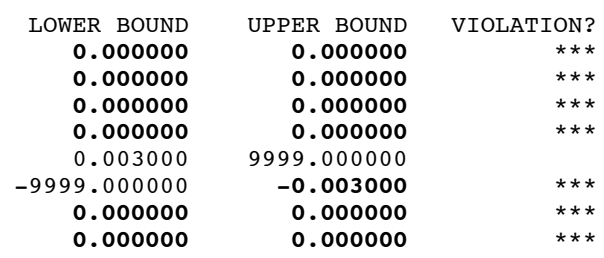

PROBLEM PARAMETERS AT DESIGN ITERATION

65

Objective function

Coef. B of flank ellipse

Coef. C of flank ellipse

Coef. F of flank ellipse

Coef. G of flank ellipse

Coef. H of flank ellipse

Total \# objective calculations

Normal termination.

OPTIMIZE: CPU secs. for this run:
$3 \quad 7.5292501222252 \mathrm{E}-01$ $7-1.1957246451232 \mathrm{E}-02$
$42.2759428039880 \mathrm{E}+00$ $8-8.7439076377836 \mathrm{E}-02$

\section{Configuration $\mathrm{X}+$ Outline}

The goal of Configuration $\mathrm{X}+$ was to match Configuration $\mathrm{X}$ as well as possible while enforcing curvature continuity everywhere, mainly to reduce the heating spike at the shoulder. Two tilted ellipses were blended 
numerically with a variation of the process described for Configuration $Z$. The only objective function implemented was to minimize (or maximize) the $x$ coordinate of the maximum diameter point $U$ on the shoulder. Since the circular shoulder of Configuration $\mathrm{X}$ is in the forward position of the simple spherical-section, $x_{U}$ was minimized, although in practice $x_{U}$ was also an optimization variable that encountered its lower bound of $0.565 \mathrm{~m}$ and stayed there.

The nose ellipse had only three coefficients to solve for (since it had to be vertical at the origin), while the outer ellipse blending with it at some point $T\left(x_{T}, y_{T}\right)$ had the maximum of five coefficients, making 11 optimization variables total. There were 10 nonlinear constraints: two conditions each for the nose and shoulder conics to be ellipses, one for the nose ellipse to pass through $T$, two for the shoulder ellipse to pass through $T$ and $U$, one for it to have zero slope at $U$, and two for matched first and second derivatives at $T$.

Since the shoulder was no longer a circle, a heuristic bridge to a notional aft body cone ( $35^{\circ}$ angle) was constructed from point $U$ by means of a cubic defined by two points and two slopes. Matching the aft segment's curvature to that of the shoulder ellipse at $U$ was not attempted and not necessarily desirable anyway.

A sequence of optimizations was actually performed with adjustments to the constraint bounds and/or variable starting guesses made in order to nudge the optimized result close to Configuration X (as determined graphicallysee Fig. A2) while achieving tight convergence to each optimum.

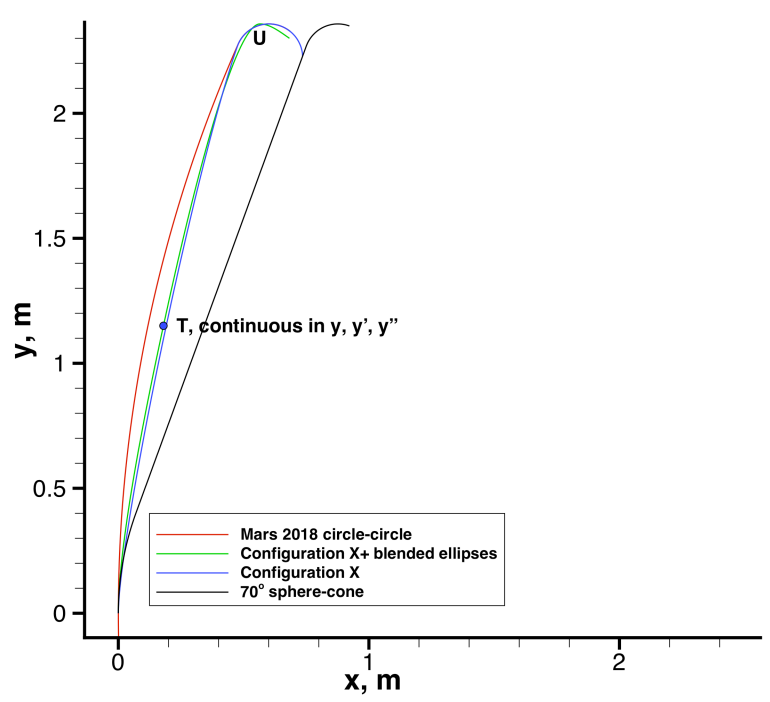

Figure A2. Comparison of Configuration $X+$ fit (ellipse-ellipse) with Configuration $X$ (ellipse-circle) and the traditional shapes.

It is hoped that the relative ease of implementation of the constrained optimization is apparent from the preceding discussion. The primary advantages of the scheme are: (1) there are no awkward solutions of nonlinear equations; (2) the entire input scheme is table driven (seen in the input listing); and (3) it is possible to satisfy specified constraints to any desired accuracy. The discussion could prove useful to any future shape optimization studies.

\section{Acknowledgments}

The authors were supported by Contract NNA10DE12C to ERC, Inc. from the Entry Systems and Technology Division at NASA Ames Research Center, and are thankful to Tom Rivellini, Ravi Prakash (both JPL), and Chun Tang (NASA ARC) for expressing interest in this work. Thanks are also due to Joseph Garcia (ARC) for using engineering tools, currently being integrated within an optimization framework at NASA Ames Research Center, to alert the authors about the performance of Configuration X. 


\section{References}

${ }^{1}$ Braun, R D, and Manning, R M, "Mars Exploration Entry, Descent, and Landing Challenges," Journal of Spacecraft and Rockets, Vol. 44, No. 2, pp. 310-323, March-April 2007.

2"Planetary Mission Entry Vehicles: A Quick Reference Guide, v3.0," C. B. Davies and M. Arcadi (compilers), NASA/SP2006-3401, 2006.

${ }^{3}$ Steltzner, A, "Mars Science Laboratory Entry, Descent and Landing System," 2006 IEEE Aerospace Conference, Big Sky, MT, Inst. of Electrical and Electronics Engineers Paper 1497, March 2006.

${ }^{4}$ Edquist, K T, Dyakonov, A A, Wright, M J, and Tang, C Y, “Aerothermodynamic Design of the Mars Science Laboratory Heatshield," AIAA Paper 2009-4075, June 2009.

${ }^{5}$ Driver, D, Carballo, J, Beck, R, Prabhu, D, Santos, J, Cassell, A, Skokova, K, Tang, C, Hwang, H, Slimko, E, Willcockson, W, Songer, J, "Arc Jet Testing in a Shear Environment for Mars Science Laboratory Thermal Protection System," AIAA-20094230 .

${ }^{6}$ Beck, R A S, Driver, D M, Wright, M J, Laub, B, Hwang, H H, Slimko, E M, Edquist, K T, Sepka, S A, Willcockson, W H, and Thames, T D, "Development of the Mars Science Laboratory Heatshield Thermal Protection System," AIAA Paper 20094229, June 2009.

${ }^{7}$ Cheatwood, F. M. and Gnoffo, P. A., "Users Manual for the Langley Aerothermodynamic Upwind Algorithm (LAURA)," NASA TM-4674, April 1996.

${ }^{8}$ Wright, M J,White, T, and Mangini, N, "Data-Parallel Line Relaxation Methods (DPLR) Code User Manual Acadia-Version 4.01.1," NASA/TM-2009-215388, October 2009.

"Anon., "NASA's Exploration Systems Architecture Study - Final Report," NASA/TM-2005-214062, November 2005.

${ }^{10}$ Brown, J L, Garcia, J, Kinney, D, and Prabhu, D, “An Asymmetric Capsule Vehicle Geometry Study for CEV," AIAA2007-604, January 2007.

${ }^{11}$ Baldwin, B. S. and Lomax, H., "Thin Layer Approximation and Algebraic Model for Separated Turbulent Flows," AIAA Paper 78-257, Jan. 1978

${ }^{12}$ Cheatwood, F. M. and Thompson, R. A., "The Addition of Algebraic Turbulence Modeling to Program LAURA," NASA TM-107758, 1993.

${ }^{13}$ Degani, D. and Schiff, L. B., "Computation of Supersonic Viscous Flows Around Pointed Bodies at Large Incidence," AIAA Paper 83-0034, Jan. 1983.

${ }^{14}$ Hirschel, E. E., Basics of aerothermodynamics. AIAA Progress in Astronautics and Aeronautics (Paul Zerchan, Editor-inChief), Vol. 206, AIAA, 2004.

${ }^{15}$ Prabhu, D. K., "System design contraints - trajectory aerothermal environments," in Hypersonic Entry and Cruise Vehicles, Vol. 2. VKI LS 2008, edited by N. N. Mansour, T. Magin, P. Moin, and O. Chazot, June-July 2008.

${ }^{16}$ Sutton, K. S., and Graves, Jr., R. A., "A General Stagnation-Point Convective-Heating Equation for Arbitrary Gas Mixtures,” NASA TR R-376, November 1971.

${ }^{17}$ Hollis, B. R., "Blunt-Body Entry Vehicle Aerothermodynamics: Transition and Turbulence on the CEV and MSL Configurations," AIAA Paper 2010-4984, 40 ${ }^{\text {th }}$ Fluid Dynamics Conference, 28 June - 1 July 2010, Chicago, IL, 2010.

${ }^{18}$ Wright, M. J., Olejniczak, J, Brown, J. L., Hornung, H. G., and Edquist, K. T., "Modeling of Shock Tunnel Aeroheating Data on the Mars Science Laboratory Aeroshell," Journal of Thermophysics and Heat Transfer, Vol. 20, No. 4, OctoberDecember 2006, pp. 641-651.

${ }^{19}$ Papadopoulos, P., Prabhu, D., Olynick, D., Chen, Y.-K., and Cheatwood, F.M., "CFD Code Comparisons for Mars Entry Simulations," AIAA Paper No. 98-0272, Jan. 1998

${ }^{20}$ Bose, D., Wright, M.J., and Palmer, G.E., "Uncertainty Analysis of Laminar Aeroheating Predictions for Mars Entries," Journal of Thermophysics and Heat Transfer, Vol. 20, No. 4, 2006, pp. 652-662.

${ }^{21}$ Wilder, M., Reda, D., and Prabhu, D., "Heat-Transfer Measurements on Hemispheres in Hypersonic Flight Through Air and CO2," AIAA Paper 2011-3476, 42nd Thermophysics Conference, Honolulu, Hawaii, June 2011.

${ }^{22}$ Gordon, S. G. and McBride, B. J., "Computer Program for Calculation of Complex Chemical Equilibrium Compositions and Applications, I: Analysis," NASA RP-1311, October 1994.

${ }^{23}$ Garcia, J., Brown, J. L., Kinney, D., Bowles, J., Jiang, X., Dupzyk, I., Huynh, L., and Lau, E., "Co-Optimization of Mid Lift to Drag Vehicles for Mars Atmospherics Entries," AIAA-2010-5052, June 2010.

${ }^{24}$ Piegl, L. A., The NURBS Book, $2^{\text {nd }}$ Edition. Monographs in Visual Communication, Springer, 1996.

${ }^{25}$ GRIDGEN, Pointwise, Inc., 213 South Jennings Ave., Fort Worth, TX 76104.

${ }^{26}$ Garcia, J., private communication, March 2011.

${ }^{27}$ Gill, P.E., Murray, W., Saunders, M.A., and Wright, M.H., "User's Guide for NPSOL (Version 4.0): A Fortran Package for Nonlinear Programming," Technical Report SOL 86-2, 1986, Department of Operations Research, Stanford University, Stanford, CA. 